\title{
Diffusive Propagation of Energy in a Non-acoustic Chain
}

\author{
Tomasz Komorowski \& Stefano Olla
}

\author{
Communicated by C. LE BRIS
}

\begin{abstract}
We consider a non-acoustic chain of harmonic oscillators with the dynamics perturbed by a random local exchange of momentum, such that energy and momentum are conserved. The macroscopic limits of the energy density, momentum and the curvature (or bending) of the chain satisfy a system of evolution equations. We prove that, in a diffusive space-time scaling, the curvature and momentum evolve following a linear system that corresponds to a damped EULER-BERNOULLI beam equation. The macroscopic energy density evolves following a non linear diffusive equation. In particular, the energy transfer is diffusive in this dynamics. This provides a first rigorous example of a normal diffusion of energy in a one dimensional dynamics that conserves the momentum.
\end{abstract}

\section{Introduction}

Macroscopic transport in a low dimensional system, in particular energy transport, has attracted attention in both the physics and mathematical physics literature in the latest decades. Anomalous energy transport has been observed numerically in Fermi-Pasta-Ulam (FPU) chains, with the diverging thermal conductivity [11]. Generically this anomalous superdiffusive behavior is attributed to the momentum conservation properties of the dynamics [10]. Actually one dimensional FPUtype chains have potential energy depending on the interparticle distances (that is the gradients of the particles displacements), and have three main locally conserved quantities: volume stretch, momentum and energy. These conserved (or balanced) quantities have different macroscopic space-time scalings, corresponding to different type of initial non-equilibrium behaviour. A mechanical non-equilibrium

This paper has been partially supported by the ANR-15-CE40-0020-01 Grant LSD; T. KomorowsKi acknowledges the support of the Polish National Science Center Grant UMO-2012/07/B/ST1/03320. 
initial profile due to the gradients of the tension induces a macroscopic ballistic evolution, at the hyperbolic space-time scale, governed by the Euler equations (cf. [6]). When the system approaches, or is already at a mechanical equilibrium, the temperature profile will evolve at a superdiffusive time scale.

Recent heuristic calculations based on fluctuating hydrodynamics theory [12], connect the macroscopic space-time scale of the superdiffusion of the thermal (energy) mode to the diffusive or superdiffusive fluctuations of the other conserved quantities. It turns out that this superdiffusive behavior of the energy is governed by a fractional laplacian heat equation. This picture can be mathematically rigorously proven in the case of a harmonic chain perturbed by a local random exchange of momentum, see [8,9]. In particular, it has been shown in [9] that in the models driven by the tension, there is a separation of the time evolution scales between the long modes (that evolve on a hyperbolic time scale) and the thermal short modes that evolve in a longer superdiffusive scale. In addition, from the explicit form of the macroscopic evolution appearing in these models, it is clear that this behavior is strongly dependent on a non-vanishing speed of sound. More specifically, when the speed of sound is null, there is no macroscopic evolution either at the hyperbolic or superdiffusive time scales. This suggests that the macroscopic evolution of the system should happen at a yet longer, possibly diffusive, time scale for all modes.

In the present article we investigate the harmonic chain model with the random exchange of momenta. The interaction potential depends only on the squares of the curvature (or bending) of the chain

$$
\mathfrak{K}_{x}:=-\Delta \mathfrak{q}_{x}:=2 \mathfrak{q}_{x}-\mathfrak{q}_{x-1}-\mathfrak{q}_{x+1}, \quad x \in \mathbb{Z},
$$

where $\mathfrak{q}_{x}$ are the positions of the particles. This means that its hamiltonian is formally given by

$$
\mathcal{H}(\mathfrak{K}, \mathfrak{p})=\sum_{x} \mathfrak{e}_{x}(\mathfrak{K}, \mathfrak{p}),
$$

where the energy of the oscillator $x$ is defined

$$
\mathfrak{e}_{x}(\mathfrak{K}, \mathfrak{p}):=\frac{\mathfrak{p}_{x}^{2}}{2}+\frac{\alpha \mathfrak{K}_{x}^{2}}{2} .
$$

Here $\alpha$ is a positive parameter that indicates the strength of the springs. This corresponds to a special choice of attractive nearest neighbor springs and repulsive next nearest neighbor springs. It turns out that the respective speed of sound is then null, even though the momentum is conserved by the dynamics. As the energy depends on the curvature and not on the volume, this system is tensionless, and the corresponding relevant conserved quantity, besides the energy and momentum, is the curvature and not the volume stretch.

Our first result, see Theorem 3.1 below, asserts that these three conserved quantities: the curvature, momentum and energy evolve together in the diffusive time scale. Denote by $k(t, y), p(t, y)$ and $e(t, y)$ the respective macroscopic limits of the fields corresponding to the aforementioned quantities. The evolution of the 
macroscopic curvature and momentum fields $k(t, y)$ and $p(t, y)$ is governed by the damped Euler-Bernoulli beam equations:

$$
\begin{aligned}
& \partial_{t} k(t, y)=-\Delta_{y} p(t, y), \\
& \partial_{t} p(t, y)=\Delta_{y}[\alpha k(t, y)+3 \gamma p(t, y)],
\end{aligned}
$$

where $\gamma>0$ is the intensity of the random exchange of momentum.

Defining the mechanical macroscopic energy as

$$
e_{\operatorname{mech}}(t, y)=\frac{1}{2}\left(p^{2}(t, y)+\alpha k^{2}(t, y)\right)
$$

and its thermal counterpart (or temperature profile) as

$$
e_{\mathrm{th}}(t, y)=e(t, y)-e_{\mathrm{mech}}(t, y),
$$

the evolution of the latter is given by

$$
\partial_{t} e_{\mathrm{th}}(t, y)=\left(\frac{(\sqrt{3}-1) \alpha}{2 \sqrt{3} \gamma}+3 \gamma\right) \Delta_{y} e_{\mathrm{th}}(t, y)+3 \gamma\left(\partial_{y} p(t, y)\right)^{2},
$$

see Theorem 3.2. In particular, the thermal conductivity is finite and we have a normal diffusion in this system. Notice also that because of the viscosity term, a gradient of the macroscopic velocity profile induces a local increase of the temperature.

This result puts in evidence two main differences between the present and the FPU-type models:

(i) the thermal conductivity is finite, even though the system is one dimensional and dynamics conserves the momentum. This suggests that the non-vanishing speed of sound is a necessary condition for the superdiffusion of the thermal energy;

(ii) there is no separation of the time scales between low (mechanical) and high (thermal) energy modes: all the frequencies evolve macroscopically in the diffusive time scale. Furthermore there is a continuous transfer of energy from low modes to high modes, resulting in the rise of the temperature, due to the gradients of the momentum profile.

These rigorous results on the harmonic non-acoustic chain open up the question if a similar behavior could appear in some deterministic non-linear Hamiltonian dynamics corresponding to an interaction of the type $V\left(\mathfrak{K}_{x}\right)$, where $\partial_{\mathfrak{q}_{x}} \mathcal{H}$ is a non linear function of the curvature of the chain.

Concerning our proof of the hydrodynamic limit. Since the microscopic energy currents in our system are not on the form of discrete space gradients of some function, this is a non-gradient system. Nevertheless we cannot use known techniques based on relative entropy methods (cf. for example [13]) for two reasons:

(i) lack of control of higher moments of the currents in terms of the relative entropy; 
(ii) degeneracy of the noise in the dynamics, as it acts only on the velocities.

Instead, we develop a method already used in [8], based on Wigner distributions for the energy of the acoustic chain. Thanks to the energy conservation property of the dynamics we can easily conclude (see Section 5.4) that the Wigner distributions form a compact family of elements in the weak topology of an appropriate Banach space. Our main result concerning the identification of its limit is contained in Theorem 5.1 below. The spatial energy density is a marginal of the Wigner function. We would like to highlight the fact that, in addition to proving the hydrodynamic limit of the energy functional, we are also able to identify the distribution of the macroscopic energy in the frequency mode domain, see formula (5.30). In particular the thermal energy is uniformly distributed on all modes (which is a form of local equilibrium), while the macroscopic mechanical energy is concentrated on the macroscopic low modes, see (5.30).

To show Theorem 5.1 we investigate the limit of the Laplace transforms of the Wigner distributions introduced in Section 7. The main results, dealing with the asymptotics of the Laplace-Wigner distributions, are formulated in Theorems 7.1-7.3. Having these results we are able to finish the identification of the limit of the Wigner distributions, thus ending the proof of Theorem 5.1. The proofs of the aforementioned Theorems 7.1-7.3, which are rather technical, are presented in Sections 8-10, respectively.

\section{The Dynamics}

\subsection{Non Acoustic Chain of Harmonic Oscillators}

Since in the non-acoustic chain the potential energy depends only on the bendings, see (1.1), in order to describe the configuration of the infinite chain we only need to specify $\left(\mathfrak{K}_{x}\right)_{x \in \mathbb{Z}}$, and the configurations of our dynamics will be denoted by $\left(\left(\mathfrak{p}_{x}, \mathfrak{K}_{x}\right)\right)_{x \in \mathbb{Z}} \in(\mathbb{R} \times \mathbb{R})^{\mathbb{Z}}$.

In case when no noise is present, the dynamics of the chain of oscillators can be written formally as a Hamiltonian system of differential equations

$$
\begin{aligned}
& \dot{\mathfrak{K}}_{x}(t)=-\Delta \partial_{\mathfrak{p}_{x}} \mathcal{H}(\mathfrak{p}(t), \mathfrak{K}(t)) \\
& \dot{\mathfrak{p}}_{x}(t)=-\partial_{\mathfrak{q}_{x}} \mathcal{H}(\mathfrak{p}(t), \mathfrak{K}(t)), \quad x \in \mathbb{Z},
\end{aligned}
$$

where $\Delta f(x)=f(x+1)+f(x-1)-2 f(x)$. Let also $\nabla g_{x}:=g_{x+1}-g_{x}$ and $\nabla^{*} g_{x}:=g_{x-1}-g_{x}$.

2.1.1. Energy-Momentum Conserving Noise. Following [1,2] we perturb the Hamiltonian dynamics (2.1) by introducing the random momentum exchange between the neighboring sites in such a way that the total momentum and energy of the system are conserved. This is achieved by adding to the right hand side of (2.1) a local stochastic term that conserves both $\mathfrak{p}_{x-1}^{2}+\mathfrak{p}_{x}^{2}+\mathfrak{p}_{x+1}^{2}$ and $\mathfrak{p}_{x-1}+\mathfrak{p}_{x}+\mathfrak{p}_{x+1}$. 
The respective stochastic differential equations can be written as

$$
\begin{aligned}
\mathrm{d} \mathfrak{K}_{x}(t)= & -\Delta \mathfrak{p}_{x}(t) \mathrm{d} t, \\
\mathrm{~d} \mathfrak{p}_{x}(t)= & {\left[\alpha \Delta \mathfrak{K}_{x}(t)-2 \gamma \check{R} * \mathfrak{p}_{x}(t)\right] \mathrm{d} t } \\
& +\gamma^{1 / 2} \sum_{z=-1,0,1} \mathcal{Y}_{x+z} \mathfrak{p}_{x}(t) \mathrm{d} w_{x+z}(t), \quad x \in \mathbb{Z},
\end{aligned}
$$

with the parameter $\gamma>0$ that indicates the strength of the noise in the system, and $\left(\mathcal{Y}_{x}\right)$ vector fields given by

$$
\mathcal{Y}_{x}:=\left(\mathfrak{p}_{x}-\mathfrak{p}_{x+1}\right) \partial_{\mathfrak{p}_{x-1}}+\left(\mathfrak{p}_{x+1}-\mathfrak{p}_{x-1}\right) \partial_{\mathfrak{p}_{x}}+\left(\mathfrak{p}_{x-1}-\mathfrak{p}_{x}\right) \partial_{\mathfrak{p}_{x+1}} .
$$

Here $\left(w_{x}(t)\right)_{t \geq 0}, x \in \mathbb{Z}$ are i.i.d. one dimensional, real valued, standard Brownian motions, over a probability space $(\Omega, \mathcal{F}, \mathbb{P})$. Furthermore, the field $\left(\check{R}_{x}\right)$ is defined as follows: $\check{R}_{x}:=\Delta \check{R}_{x}^{(0)}$, where

$$
\check{R}_{x}^{(0)}:=\left\{\begin{aligned}
-1, & x=0 \\
-\frac{1}{4}, & x= \pm 1 \\
0, & \text { if otherwise }
\end{aligned}\right.
$$

As a result we obtain $\check{R}_{0}=3 / 2, \check{R}_{ \pm 1}=-1 / 2, \check{R}_{ \pm 2}=-1 / 4$ and $\check{R}_{x}=0$, if $|x| \geq 3$. In addition, for any two fields $\left(a_{x}\right),\left(b_{x}\right)$ we define their convolution by letting $(a * b)_{x}:=\sum_{y \in \mathbb{Z}} a_{x-y} b_{y}$. This definition makes sense for example in the case when one of the fields is of compact support (as for example $\left(\check{R}_{x}\right)$ here).

We can rewrite the system (2.2)

$$
\begin{aligned}
\mathrm{d} \mathfrak{K}_{x}(t)= & -\Delta \mathfrak{p}_{x}(t) \mathrm{d} t \\
\mathrm{~d} \mathfrak{p}_{x}(t)= & {\left[\alpha \Delta \mathfrak{K}_{x}(t)+2 \gamma \Delta\left(\check{R}^{(0)} * \mathfrak{p}(t)\right)_{x}\right] \mathrm{d} t } \\
& +\gamma^{1 / 2} \sum_{z=-1,0,1}\left(\mathcal{Y}_{x+z} \mathfrak{p}_{x}(t)\right) \mathrm{d} w_{x+z}(t), \quad x \in \mathbb{Z} .
\end{aligned}
$$

Remark. The particular choice of the random exchange in the above dynamics is not important. The result can be extended to any other random mechanism of momentum exchange, as long as total energy and momentum are conserved. Most simple dynamics would be given by exchange of momentum between nearest neighbor atoms at independent exponential times.

\subsection{Stationary Gibbs Distributions}

Let $\lambda=(\beta, \bar{p}, \kappa)$, with $\beta^{-1} \geq 0$ and $\bar{p}, \kappa \in \mathbb{R}$. The product measures

$$
\begin{aligned}
\mathrm{d} \mu_{\lambda} & :=\prod_{x} \exp \left\{-\beta\left(\mathfrak{e}_{x}-\bar{p} \mathfrak{p}_{x}-\kappa \mathfrak{K}_{x}\right)-\mathcal{G}(\lambda)\right\} \mathrm{d} \mathfrak{K}_{x} \mathrm{~d} \mathfrak{p}_{x}, \\
\mathcal{G}(\boldsymbol{\lambda}) & :=\frac{1}{2} \log \left(\frac{2 \pi \beta}{\alpha}\right)+\frac{\beta\left(\bar{p}^{2}+\kappa^{2} / \alpha\right)}{2}
\end{aligned}
$$


are stationary for the dynamics defined by (2.4). In this context $\kappa$ is called the load of the chain, while as usual $\beta^{-1}$ is the temperature and $\bar{p}$ is the average momentum. When $\beta^{-1}=0$ the measure in (2.5) is, by a convention, the product of delta type measures, each concentrated at $(\kappa, \bar{p})$.

\subsection{Initial Data}

Concerning the initial data we assume that:

(A1) given $\epsilon>0$, it is distributed according to a probability measure $\mu_{\epsilon}$ on the configuration of $\left(\left(\mathfrak{K}_{x}, \mathfrak{p}_{x}\right)\right)_{x \in \mathbb{Z}}$ and satisfies

$$
\sup _{\epsilon \in(0,1]} \epsilon \sum_{x}\left\langle\mathfrak{e}_{x}\right\rangle_{\mu_{\epsilon}}<+\infty
$$

Here $\langle\cdot\rangle_{\mu_{\epsilon}}$ denotes the average with respect to $\mu_{\epsilon}$. We denote also by $\mathbb{E}_{\epsilon}$ the expectation with respect to the product measure $\mathbb{P}_{\epsilon}=\mu_{\epsilon} \otimes \mathbb{P}$.

The existence and uniqueness of a solution to (2.2) in $\ell_{2}$, with the aforementioned initial condition can be easily concluded from the standard Hilbert space theory of stochastic differential equations, see for example Chapter 6 of [4]. We assume furthermore that

(A2) the mean of the initial configuration varies on the macroscopic spatial scale:

$$
\left\langle\mathfrak{K}_{x}\right\rangle_{\mu_{\epsilon}}=\kappa(\epsilon x), \quad\left\langle\mathfrak{p}_{x}\right\rangle_{\mu_{\epsilon}}=p(\epsilon x), \quad x \in \mathbb{Z}
$$

for some functions $\kappa, p \in C_{0}^{\infty}(\mathbb{R})$. Then, the respective Fourier transforms $\hat{\kappa}$ and $\hat{p}$ belong to the Schwartz class $\mathcal{S}(\mathbb{R})$.

As for the fluctuations around the mean, we assume that their energy spectrum is uniformly $L^{r}$ integrable with respect to $\epsilon>0$ for some $r>1$. We have denoted by

$$
\hat{f}(k):=\sum_{x} f_{x} \exp \{-2 \pi i k x\}, \quad k \in \mathbb{T}
$$

the Fourier transform of a given sequence $f_{x}, x \in \mathbb{Z}$. Here $\mathbb{T}$ is the unit torus, understood as the interval $[-1 / 2,1 / 2]$ with the identified endpoints. Let

$$
\tilde{\mathfrak{K}}_{x}:=\mathfrak{K}_{x}-\left\langle\mathfrak{K}_{x}\right\rangle_{\mu_{\epsilon}}, \text { and } \tilde{\mathfrak{p}}_{x}:=\mathfrak{p}_{x}-\left\langle\mathfrak{p}_{x}\right\rangle_{\mu_{\epsilon}}, \quad x \in \mathbb{Z} .
$$

The energy spectrum is defined as:

$$
\mathcal{E}_{\epsilon}(k):=\frac{1}{2}\left[\left\langle|\hat{\tilde{\mathfrak{p}}}(k)|^{2}\right\rangle_{\mu_{\epsilon}}+\alpha\left\langle|\hat{\tilde{\mathfrak{K}}}(k)|^{2}\right\rangle_{\mu_{\epsilon}}\right], \quad k \in \mathbb{T},
$$

where $\hat{\tilde{\mathfrak{p}}}(k)$ and $\hat{\tilde{\mathfrak{K}}}(k)$ are the Fourier transforms of $\left(\tilde{\mathfrak{p}}_{x}\right)$ and $\left(\tilde{\mathfrak{K}}_{x}\right)$, respectively. Assumption (2.6) implies in particular that

$$
K_{0}=\sup _{\epsilon \in(0,1]} \epsilon \int_{\mathbb{T}} \mathcal{E}_{\epsilon}(k) \mathrm{d} k<+\infty .
$$

The announced property of the $L^{r}$ integrability of the energy spectrum can be formulated as follows: 
(A3) there exists $r>1$ such that:

$$
K_{1}:=\sup _{\epsilon \in(0,1]} \epsilon^{r} \int_{\mathbb{T}} \mathcal{E}_{\epsilon}^{r}(k) \mathrm{d} k<+\infty .
$$

Thanks to the hypothesis (2.7) we conclude that for any $G \in C_{0}^{\infty}(\mathbb{R})$ we have

$$
\begin{aligned}
\lim _{\epsilon \rightarrow 0+} \epsilon \sum_{x} G(\epsilon x)\left\langle\mathfrak{p}_{x}\right\rangle_{\mu_{\epsilon}} & =\int_{\mathbb{R}} G(y) p(y) \mathrm{d} y, \\
\lim _{\epsilon \rightarrow 0+} \epsilon \sum_{x} G(\epsilon x)\left\langle\mathfrak{K}_{x}\right\rangle_{\mu_{\epsilon}} & =\int_{\mathbb{R}} G(y) \kappa(y) \mathrm{d} y .
\end{aligned}
$$

The quantities $p(\cdot), \kappa(\cdot)$ are called the macroscopic velocity and curvature profiles. We assume furthermore that:

(A4) the following limits exist

$$
\begin{aligned}
& \lim _{\epsilon \rightarrow 0+} \epsilon \sum_{x} G(\epsilon x)\left\langle\mathfrak{p}_{x}^{2}\right\rangle_{\mu_{\epsilon}}=\int_{\mathbb{R}} G(y) p_{2}(y) \mathrm{d} y, \\
& \lim _{\epsilon \rightarrow 0+} \epsilon \sum_{x} G(\epsilon x)\left\langle\mathfrak{K}_{x}^{2}\right\rangle_{\mu_{\epsilon}}=\int_{\mathbb{R}} G(y) \kappa_{2}(y) \mathrm{d} y, \\
& \lim _{\epsilon \rightarrow 0+} \epsilon \sum_{x} G(\epsilon x)\left\langle\mathfrak{K}_{x} \mathfrak{p}_{x}\right\rangle_{\mu_{\epsilon}}=\int_{\mathbb{R}} G(y) j(y) \mathrm{d} y,
\end{aligned}
$$

for any $G \in C_{0}^{\infty}(\mathbb{R})$. Here $j(\cdot), p_{2}(\cdot), \kappa_{2}(\cdot)$ are some functions belonging to $C_{0}^{\infty}(\mathbb{R})$.

As a consequence, we conclude that the limit

$$
\lim _{\epsilon \rightarrow 0+} \epsilon \sum_{x} G(\epsilon x)\left\langle\mathfrak{e}_{x}\right\rangle_{\mu_{\epsilon}}=\int_{\mathbb{R}} G(y) e(y) \mathrm{d} y
$$

also exists for any $G \in C_{0}^{\infty}(\mathbb{R})$. Here $e(y)$ - the macroscopic energy profile - is given by

$$
e(y)=\frac{1}{2}\left(p_{2}(y)+\alpha \kappa_{2}(y)\right) .
$$

Remark. An important example of initial distributions that satisfy the above conditions is provided by local Gibbs measures (see Section 9.2.5 of [9]). That is inhomogeneous product probability measures of the type

$$
\prod_{x \in \mathbb{Z}} \exp \left\{-\beta_{x}\left(\mathfrak{e}_{x}-p_{x} \mathfrak{p}_{x}-\kappa_{x} \mathfrak{K}_{x}\right)-\mathcal{G}\left(\lambda_{x}\right)\right\} \mathrm{d} \mathfrak{K}_{x} d \mathfrak{p}_{x}
$$

Here the vector $\lambda_{x}=\left(\beta_{x}, p_{x}, \kappa_{x}\right)$ is given by $\lambda_{x}:=\lambda(\epsilon x)$, where $\lambda(x):=$ $(\beta(x), p(x), \kappa(x))$ and the functions $\beta^{-1}(\cdot), p(\cdot), \kappa(\cdot)$ belong to $C_{0}^{\infty}(\mathbb{R})$. On the sites where $\beta(\epsilon x)^{-1}=0$, we let the corresponding exponential factor in (2.17) be a delta distribution concentrated at the point $(\kappa(\epsilon x), p(\epsilon x))$. In this case

$$
\begin{aligned}
& j(y)=\kappa(y) p(y), \quad p_{2}(y)=p^{2}(y)+\beta^{-1}(y), \\
& \kappa_{2}(y)=\alpha^{-1}\left(\alpha^{-1} \kappa^{2}(y)+\beta^{-1}(y)\right) .
\end{aligned}
$$




\section{Formulation of the Main Results}

Suppose that $p(t, y), \kappa(t, y)$ satisfy the following Cauchy problem

$$
\begin{aligned}
& \partial_{t} \kappa(t, y)=-\Delta_{y} p(t, y), \\
& \partial_{t} p(t, y)=\alpha \Delta_{y} \kappa(t, y)+3 \gamma \Delta_{y} p(t, y), \\
& p(0, y)=p(y), \quad \kappa(0, y)=\kappa(y),
\end{aligned}
$$

with $p(\cdot), \kappa(\cdot)$ given by $(2.13)$.

Remark. The above system is uniformly parabolic in the Petrovskii sense for any $\gamma>0$, see for example p. 8 of [5]. The solution exists and is uniquely determined by the fact that its Fourier transform $[\hat{p}(t, \eta), \hat{\kappa}(t, \eta)]$ satisfies the system of ordinary differential equations

$$
\begin{aligned}
& \frac{\mathrm{d}}{\mathrm{d} t} \hat{\kappa}(t, \eta)=|\eta|^{2} \hat{p}(t, \eta), \\
& \frac{\mathrm{d}}{\mathrm{d} t} \hat{p}(t, \eta)=-\alpha|\eta|^{2} \hat{\kappa}(t, \eta)-3 \gamma|\eta|^{2} \hat{p}(t, \eta), \\
& \hat{p}(0, \eta)=\hat{p}(\eta), \quad \hat{\kappa}(0, \eta)=\hat{\kappa}(\eta),
\end{aligned}
$$

see pp. 44-47 in [5].

Our first result concerns the evolution of the macroscopic profiles of the velocity and curvature.

Theorem 3.1. Under the assumptions (A1)-(A4) for any $G \in C_{0}^{\infty}(\mathbb{R})$ and $t \geq 0$ we have

$$
\begin{aligned}
& \lim _{\epsilon \rightarrow 0+} \epsilon \sum_{x} G(\epsilon x) \mathbb{E}_{\epsilon} \mathfrak{p}_{x}\left(\frac{t}{\epsilon^{2}}\right)=\int_{\mathbb{R}} G(y) p(t, y) \mathrm{d} y, \\
& \lim _{\epsilon \rightarrow 0+} \epsilon \sum_{x} G(\epsilon x) \mathbb{E}_{\epsilon} \mathfrak{K}_{x}\left(\frac{t}{\epsilon^{2}}\right)=\int_{\mathbb{R}} G(y) \kappa(t, y) \mathrm{d} y,
\end{aligned}
$$

where $p(t, y)$ and $\kappa(t, y)$ is the solution of (3.1).

The proof of this result is fairly standard and we show it in Section 11. Define the macroscopic profile of the mechanical energy of the chain by

$$
e_{\text {mech }}(t, y):=\frac{1}{2}\left(p^{2}(t, y)+\alpha \kappa^{2}(t, y)\right),
$$

with $e_{\text {mech }}^{(0)}(y):=e_{\text {mech }}(0, y)$. Comparing with the energy profile at $t=0$, given by (2.16), we conclude that the residual energy, called the initial thermal energy (or temperature) profile, satisfies

$$
e_{\mathrm{th}}^{(0)}(y):=e(y)-e_{\mathrm{mech}}^{(0)}(y) \geq 0 .
$$

Concerning the evolution of the energy profile we have the following result. 
Theorem 3.2. Under the assumptions (A1)-(A4) about the initial data the limit

$$
\lim _{\epsilon \rightarrow 0+} \epsilon \sum_{x} \int_{0}^{+\infty} G(t, \epsilon x) \mathbb{E}_{\epsilon} \mathfrak{e}_{x}\left(\frac{t}{\epsilon^{2}}\right) \mathrm{d} t=\int_{0}^{+\infty} \int_{\mathbb{R}} G(t, y) e(t, y) \mathrm{d} t \mathrm{~d} y
$$

exists for any $G \in C_{0}^{\infty}([0,+\infty) \times \mathbb{R})$. In addition, we have

$$
e(t, y)=e_{\mathrm{th}}(t, y)+e_{\mathrm{mech}}(t, y)
$$

where $e_{\text {mech }}(t, y)$ is given by (3.4), while the thermal energy (temperature) $e_{\mathrm{th}}(t, y)$ is the solution of the following Cauchy problem:

$$
\begin{aligned}
& \partial_{t} e_{\mathrm{th}}(t, y)=\hat{c} \partial_{y}^{2} e_{\mathrm{th}}(t, y)+3 \gamma\left(\partial_{y} p\right)^{2}(t, y), \\
& e_{\mathrm{th}}(0, y)=e_{\mathrm{th}}^{(0)}(y) .
\end{aligned}
$$

The diffusivity coefficient equals (see formula (9.21) below)

$$
\hat{c}:=\frac{(\sqrt{3}-1) \alpha}{2 \gamma \sqrt{3}}+3 \gamma .
$$

Remark 3.3. Notice that the gradient of the macroscopic momentum $p(t, y)$ appearing in (3.7) causes a local increase of the temperature. It is also straightforward to understand the appearance of this term in the aforementioned equation. Consider for simplicity the case $\alpha=0$. The dynamics is constituted then only by the random exchanges of the momentum. The conserved quantities that evolve macroscopically are the momentum $\mathfrak{p}_{x}$ and the kinetic energy $\mathfrak{p}_{x}^{2} / 2$. The corresponding macroscopic equations are

$$
\begin{aligned}
& \partial_{t} p=3 \gamma \partial_{y}^{2} p \\
& \partial_{t} e=3 \gamma \partial_{y}^{2} e .
\end{aligned}
$$

These can be proven easily since the microscopic dynamics is of gradient type. It follows that the macroscopic equation for the temperature field, defined by $e_{\text {th }}=$ $e-p^{2} / 2$, is given by

$$
\partial_{t} e_{\mathrm{th}}(t, y)=3 \gamma\left\{\partial_{y}^{2} e_{\mathrm{th}}(t, y)+\left(\partial_{y} p\right)^{2}(t, y)\right\} .
$$

The interaction $\alpha$ affects the thermal diffusivity, but does not influence the nonlinearity appearing in the evolution of the temperature profile.

\section{Some Basic Notation}

To abbreviate our notation, we write

$$
\mathfrak{s}(k):=\sin (\pi k) \quad \text { and } \quad \mathfrak{c}(k):=\cos (\pi k), \quad k \in \mathbb{T} .
$$


Let $\ell^{2}$ be the space of all complex valued sequences $\left(f_{x}\right)_{x \in \mathbb{Z}}$, equipped with the norm $\|f\|_{\ell^{2}}^{2}:=\sum_{x}\left|f_{x}\right|^{2}$. Obviously $\hat{f}$ belongs to $L^{2}(\mathbb{T})$ - the space of all complex valued functions equipped with the norm $\|f\|_{L^{2}(\mathbb{T})}:=\langle\hat{f}, \hat{f}\rangle_{L^{2}(\mathbb{T})}^{1 / 2}$, where

$$
\langle\hat{f}, \hat{g}\rangle_{L^{2}(\mathbb{T})}:=\int_{\mathbb{T}} \hat{f}(k) \hat{g}^{*}(k) \mathrm{d} k, \quad \hat{f}, \hat{g} \in L^{2}(\mathbb{T}) .
$$

Given a set $A$ and two functions $f, g: A \rightarrow \mathbb{R}_{+}$we say that $f(x) \asymp g(x)$, $x \in A$ if there exists $C>1$ such that

$$
\frac{f(x)}{C} \leq g(x) \leq C f(x), \quad \forall x \in A .
$$

We write $g(x) \preceq f(x)$, when only the upper bound on $g$ is satisfied.

Denote by $\mathcal{S}$ the set of functions $J: \mathbb{R} \times \mathbb{T} \rightarrow \mathbb{C}$ that are of $C^{\infty}$ class and such that for any integers $l, m, n$ we have

$$
\sup _{y \in \mathbb{R}, k \in \mathbb{T}}\left(1+y^{2}\right)^{n}\left|\partial_{y}^{l} \partial_{k}^{m} J(y, k)\right|<+\infty .
$$

For $J \in \mathcal{S}$ we let $\hat{J}$ be its Fourier transform in the first variable, that is

$$
\hat{J}(\eta, k):=\int_{\mathbb{R}} e^{-2 \pi i y \eta} J(y, k) \mathrm{d} y, \quad(\eta, k) \in \mathbb{R} \times \mathbb{T} .
$$

For any $M>0$ let $\mathcal{A}_{M}$ be the completion of $\mathcal{S}$ in the norm

$$
\|J\|_{\mathcal{A}_{M}}:=\sup _{|\eta| \leq M}\left(\int_{\mathbb{T}}|\hat{J}(\eta, k)| \mathrm{d} k\right) .
$$

We drop the subscript from the notation if $M=+\infty$. Let $\mathcal{A}^{\prime}$ and $\mathcal{A}_{M}^{\prime}$ be the respective topological dual spaces of $\mathcal{A}$ and $\mathcal{A}_{M}$. The space $\mathcal{A}_{M}^{\prime}$ is made of equivalence classes of measurable functions, for which the pseudo-norm

$$
\|J\|_{\mathcal{A}_{M}^{\prime}}:=\int_{|\eta| \leq M}\left(\sup _{k \in \mathbb{T}}|\hat{J}(\eta, k)|\right) \mathrm{d} \eta
$$

is finite.

\section{Wigner Function and its Evolution}

\subsection{The Wave Function}

The wave function corresponding to the configuration $\left(\left(\mathfrak{p}_{x}, \mathfrak{K}_{x}\right)\right)_{x \in \mathbb{Z}}$ is defined as

$$
\psi_{x}:=\sqrt{\alpha} \mathfrak{K}_{x}+i \mathfrak{p}_{x}, \quad x \in \mathbb{Z}
$$

Its Fourier transform is given by

$$
\hat{\psi}(k)=\sqrt{\alpha} \hat{\mathfrak{K}}(k)+i \hat{\mathfrak{p}}(k), \quad k \in \mathbb{T} .
$$


The energy and its spectrum (2.10) can be written as

$$
\mathfrak{e}_{x}=\frac{1}{2}\left|\psi_{x}\right|^{2}, \quad x \in \mathbb{Z} \text { and } \mathcal{E}_{\epsilon}(k)=\frac{1}{2}\left\langle|\hat{\psi}(k)|^{2}\right\rangle_{\mu_{\epsilon}}, \quad k \in \mathbb{T} .
$$

Using the decomposition into the macroscopic profile and the fluctuation part, see (2.9), we can write

$$
\psi_{x}=\phi(\epsilon x)+\tilde{\psi}_{x}^{(\epsilon)}, \quad x \in \mathbb{Z}
$$

where

$$
\phi(y):=\sqrt{\alpha} \kappa(y)+i p(y) \text { and } \tilde{\psi}_{x}^{(\epsilon)}:=\sqrt{\alpha} \tilde{\mathfrak{K}}_{x}+i \tilde{\mathfrak{p}}_{x}
$$

are the wave functions corresponding to the macroscopic profile and the fluctuation part, respectively.

\subsection{Wigner Functions}

By the Wigner functions corresponding to the wave function field $\left(\psi_{x}\right)_{x \in \mathbb{Z}}$ we understand four tempered distributions $W_{\epsilon, \pm}, Y_{\epsilon, \pm}$ that we often write together in the form of a vector

$$
\mathfrak{W}_{\epsilon}^{T}:=\left[W_{\epsilon,+}, Y_{\epsilon,+}, Y_{\epsilon,-}, W_{\epsilon,-}\right],
$$

where

$$
\left\langle W_{\epsilon, \pm}, J\right\rangle:=\int_{\mathbb{R} \times \mathbb{T}} \widehat{W}_{\epsilon, \pm}(\eta, k) \hat{J}^{*}(\eta, k) \mathrm{d} \eta \mathrm{d} k
$$

and

$$
\left\langle Y_{\epsilon, \pm}, J\right\rangle:=\int_{\mathbb{R} \times \mathbb{T}} \widehat{Y}_{\epsilon, \pm}(\eta, k) \hat{J}^{*}(\eta, k) \mathrm{d} \eta \mathrm{d} k
$$

for any $J \in \mathcal{A}$. Here $\widehat{W}_{\epsilon, \pm}(\eta, k)$ and $\widehat{Y}_{\epsilon, \pm}(\eta, k)$ —called the Fourier-Wigner functionsare given by

$$
\begin{aligned}
\widehat{W}_{\epsilon,+}(\eta, k) & :=\frac{\epsilon}{2}\left\langle(\hat{\psi})^{*}\left(k-\frac{\epsilon \eta}{2}\right) \hat{\psi}\left(k+\frac{\epsilon \eta}{2}\right)\right\rangle_{\mu_{\epsilon}}, \\
\widehat{Y}_{\epsilon,+}(\eta, k) & :=\frac{\epsilon}{2}\left\langle\hat{\psi}\left(-k+\frac{\epsilon \eta}{2}\right) \hat{\psi}\left(k+\frac{\epsilon \eta}{2}\right)\right\rangle_{\mu_{\epsilon}}, \\
\widehat{Y}_{\epsilon,-}(\eta, k) & :=\widehat{Y}_{\epsilon,+}^{*}(-\eta, k), \quad \widehat{W}_{\epsilon,-}(\eta, k):=\widehat{W}_{\epsilon,+}(\eta,-k) .
\end{aligned}
$$

For any $J \in \mathcal{A}$ we can write

$$
\left|\left\langle W_{\epsilon,+}, J\right\rangle\right| \leq \frac{\epsilon}{2}\|J\|_{\mathcal{A}} \sup _{\eta} \int_{\mathbb{T}}\left|\left\langle\hat{\psi}\left(k-\frac{\epsilon \eta}{2}\right) \hat{\psi}\left(k+\frac{\epsilon \eta}{2}\right)\right\rangle_{\mu_{\epsilon}}\right| \mathrm{d} k .
$$

Using the Cauchy-Schwarz inequality and (2.11) we get

$$
\sup _{\epsilon>0} \sum_{l= \pm}\left(\left\|Y_{\epsilon, l}\right\|_{\mathcal{A}^{\prime}}+\left\|W_{\epsilon, l}\right\|_{\mathcal{A}^{\prime}}\right) \leq 4 K_{0}
$$


By Plancherel's identity we obtain that for any $J \in \mathcal{S}$

$$
\left\langle W_{\epsilon, \pm}, J\right\rangle=\frac{\epsilon}{2} \sum_{x, x^{\prime} \in \mathbb{Z}}\left\langle\left(\psi_{x^{\prime}}\right)^{*} \psi_{x}\right\rangle_{\mu_{\epsilon}} \int_{\mathbb{T}} e^{2 \pi i\left(x^{\prime}-x\right) k} J^{*}\left(\frac{\epsilon}{2}\left(x+x^{\prime}\right), \pm k\right) \mathrm{d} k .
$$

Substituting $J(y, k) \equiv J(y)$, we obtain

$$
\left\langle W_{\epsilon, \pm}, J\right\rangle=\epsilon \sum_{x}\left\langle\mathfrak{e}_{x}\right\rangle_{\mu_{\epsilon}} J^{*}(\epsilon x)
$$

and likewise

$$
\left\langle Y_{\epsilon, \pm}, J\right\rangle=\epsilon \sum_{x}\left\langle\mathfrak{l}_{x} \pm i \sqrt{\alpha} \mathfrak{j}_{x}\right\rangle_{\mu_{\epsilon}} J^{*}(\epsilon x)
$$

where

$$
\mathfrak{l}_{x}:=\frac{1}{2}\left(\alpha \mathfrak{K}_{x}^{2}-\mathfrak{p}_{x}^{2}\right), \quad \mathfrak{j}_{x}:=\mathfrak{K}_{x} \mathfrak{p}_{x}, \quad x \in \mathbb{Z}
$$

Using the decomposition of the wave function into its mean, following a macroscopic profile $\phi(\cdot)$, and the fluctuation part $\left\{\tilde{\psi}_{x}^{(\epsilon)}, x \in \mathbb{Z}\right\}$, see (5.4), we can correspondingly decompose the vector of the Wigner functions. Namely,

$$
\mathfrak{W}_{\epsilon}=\overline{\mathfrak{W}}_{\epsilon}+\widetilde{\mathfrak{W}}_{\epsilon},
$$

where the Fourier-Wigner function corresponding to these wave functions shall be denoted by

$$
\overline{\mathfrak{W}}_{\epsilon}^{T}:=\left[\bar{W}_{\epsilon,+}, \bar{Y}_{\epsilon,+}, \bar{Y}_{\epsilon,-}, \bar{W}_{\epsilon,-}\right]
$$

and

$$
\widetilde{\mathfrak{W}}_{\epsilon}^{T}:=\left[\widetilde{W}_{\epsilon,+}, \widetilde{Y}_{\epsilon,+}, \widetilde{Y}_{\epsilon,-}, \widetilde{W}_{\epsilon,-}\right]
$$

We let

$$
\begin{aligned}
\left\langle\bar{W}_{\epsilon, \pm}, J\right\rangle & :=\int_{\mathbb{R} \times \mathbb{T}} \widehat{\bar{W}}_{\epsilon, \pm}(\eta, k) J^{*}(\eta, k) \mathrm{d} \eta \mathrm{d} k, \\
\left\langle\widetilde{W}_{\epsilon, \pm}, J\right\rangle & :=\int_{\mathbb{R} \times \mathbb{T}} \widehat{\widetilde{W}}_{\epsilon, \pm}(\eta, k) J^{*}(\eta, k) \mathrm{d} \eta \mathrm{d} k,
\end{aligned}
$$

where, using the Poisson summation formula, we have defined

$$
\begin{aligned}
& \widehat{\bar{W}}_{\epsilon, \pm}(\eta, k)=\frac{1}{2 \epsilon} \sum_{x, x^{\prime}} \hat{\phi}^{*}\left(\frac{ \pm k+x}{\epsilon}-\frac{\eta}{2}\right) \hat{\phi}\left(\frac{ \pm k+x^{\prime}}{\epsilon}+\frac{\eta}{2}\right), \\
& \widehat{\widetilde{W}}_{\epsilon, \pm}(\eta, k)=\frac{\epsilon}{2}\left\langle\left(\hat{\tilde{\psi}}^{(\epsilon)}\right)^{*}\left( \pm k-\frac{\epsilon \eta}{2}\right) \hat{\tilde{\psi}}^{(\epsilon)}\left( \pm k+\frac{\epsilon \eta}{2}\right)\right\rangle_{\mu_{\epsilon}} .
\end{aligned}
$$

The formulas for $\left\langle\bar{Y}_{\epsilon, \pm}, J\right\rangle$ and $\left\langle\widetilde{Y}_{\epsilon, \pm}, J\right\rangle$ are constructed analogously using the respective Fourier-Wigner functions. Notice that for small $\epsilon$ the expression above of $\widehat{W}_{\epsilon, \pm}$ is well approximated by the more natural definition:

$$
\widehat{\bar{W}}_{\epsilon, \pm}(\eta, k) \sim \frac{\epsilon}{2} \hat{\phi}^{*}\left(\frac{ \pm k}{\epsilon}-\frac{\eta}{2}\right) \hat{\phi}\left(\frac{ \pm k}{\epsilon}+\frac{\eta}{2}\right) .
$$


As a consequence of assumption (2.14) we conclude that for functions $J(y, k)=$ $J(y)$ :

$$
\begin{aligned}
& \lim _{\epsilon \rightarrow 0+}\left\langle W_{\epsilon, \pm}, J\right\rangle=\int_{\mathbb{R}} e(y) J^{*}(y) \mathrm{d} y \\
& \lim _{\epsilon \rightarrow 0+}\left\langle Y_{\epsilon, \pm}, J\right\rangle=\int_{\mathbb{R}}(l(y) \pm i \sqrt{\alpha} j(y)) J^{*}(y) \mathrm{d} y
\end{aligned}
$$

and

$$
l(y):=\frac{1}{2}\left(\alpha \kappa_{2}(y)-p_{2}(y)\right),
$$

with $j(\cdot), \kappa_{2}(\cdot)$ and $p_{2}(\cdot)$ given by (2.14). A simple calculation also shows that

$$
\begin{aligned}
\lim _{\epsilon \rightarrow 0+}\left\langle\bar{W}_{\epsilon, \pm}, J\right\rangle & =\left\langle\bar{W}_{ \pm}, J\right\rangle:=\frac{1}{2} \int_{\mathbb{R}^{2}} \hat{\phi}^{*}\left( \pm h-\frac{\eta}{2}\right) \hat{\phi}\left( \pm h+\frac{\eta}{2}\right) \hat{J}^{*}(\eta, 0) \mathrm{d} \eta \mathrm{d} h \\
& =\frac{1}{2} \int_{\mathbb{R}^{2}}\left|\phi^{*}(y)\right|^{2} e^{-2 \pi i y \eta} \hat{J}^{*}(\eta, 0) \mathrm{d} \eta \mathrm{d} y .
\end{aligned}
$$

Thus,

$$
\bar{W}_{ \pm}(\mathrm{d} y, \mathrm{~d} k)=\frac{1}{2}|\phi(y)|^{2} \delta_{0}(\mathrm{~d} k) \mathrm{d} y .
$$

One can also easily check that

$$
\bar{Y}_{+}(\mathrm{d} y, \mathrm{~d} k)=\frac{1}{2} \phi^{2}(y) \delta_{0}(\mathrm{~d} k) \mathrm{d} y \quad \text { and } \quad \bar{Y}_{-}(\mathrm{d} y, \mathrm{~d} k)=\frac{1}{2}\left[\phi^{*}(y)\right]^{2} \delta_{0}(\mathrm{~d} k) \mathrm{d} y .
$$

We denote the respective vector $\overline{\mathfrak{W}}^{T}:=\left[\bar{W}_{+}, \bar{Y}_{+}, \bar{Y}_{-}, \bar{W}_{+}\right]$.

\subsection{Evolution of the Wave Function}

Adjusted to the macroscopic time, we can define the wave function corresponding to the configuration at time $t / \epsilon^{2}$

$$
\psi_{x}^{(\epsilon)}(t):=\sqrt{\alpha} \mathfrak{K}_{x}\left(\epsilon^{-2} t\right)+i \mathfrak{p}_{x}\left(\epsilon^{-2} t\right), \quad x \in \mathbb{Z},
$$

where $\left(\mathfrak{p}_{x}(t), \mathfrak{K}_{x}(t)\right)_{x \in \mathbb{Z}}$ satisfies (2.2). Its Fourier transform

$$
\hat{\psi}^{(\epsilon)}(t, k)=\sqrt{\alpha} \hat{\mathfrak{K}}\left(\frac{t}{\epsilon^{2}}, k\right)+i \hat{\mathfrak{p}}\left(\frac{t}{\epsilon^{2}}, k\right), \quad k \in \mathbb{T},
$$

is the unique solution of the Itô stochastic differential equation, understood in the mild sense (see for example Theorem 7.4 of [4])

$$
\begin{aligned}
\mathrm{d} \hat{\psi}^{(\epsilon)}(t, k)= & \left\{\frac{-i \omega(k)}{\epsilon^{2}} \hat{\psi}^{(\epsilon)}(t, k)-\frac{\gamma R(k)}{\epsilon^{2}}\left[\hat{\psi}^{(\epsilon)}(t, k)-\left(\hat{\psi}^{(\epsilon)}\right)^{*}(t,-k)\right]\right\} \mathrm{d} t \\
& +\frac{i \gamma^{1 / 2}}{\epsilon} \int_{\mathbb{T}} r\left(k, k^{\prime}\right)\left[\hat{\psi}^{(\epsilon)}\left(t, k-k^{\prime}\right)-\left(\hat{\psi}^{(\epsilon)}\right)^{*}\left(t, k^{\prime}-k\right)\right] B\left(\mathrm{~d} t, \mathrm{~d} k^{\prime}\right),
\end{aligned}
$$


where $\hat{\psi}^{(\epsilon)}(0) \in L^{2}(\mathbb{T})$,

$$
\omega(k):=2 \sqrt{\alpha} \mathfrak{s}^{2}(k), \quad k \in \mathbb{T}
$$

is the dispersion relation, and

$$
R(k):=2 \mathfrak{s}^{2}(k)\left[1+2 \mathfrak{c}^{2}(k)\right]=2 \mathfrak{s}^{2}(k)+\mathfrak{s}^{2}(2 k) .
$$

Here

$$
r\left(k, k^{\prime}\right):=4 \mathfrak{s}(k) \mathfrak{s}\left(k-k^{\prime}\right) \mathfrak{s}\left(2 k-k^{\prime}\right), \quad k, k^{\prime} \in \mathbb{T} .
$$

The process $B(\mathrm{~d} t, \mathrm{~d} k)$ is a space-time Gaussian white noise, that is

$$
\mathbb{E}\left[B(\mathrm{~d} t, \mathrm{~d} k) B^{*}\left(\mathrm{~d} s, \mathrm{~d} k^{\prime}\right)\right]=\delta(t-s) \delta\left(k-k^{\prime}\right) \mathrm{d} t \mathrm{~d} s \mathrm{~d} k \mathrm{~d} k^{\prime} .
$$

Since the total energy of the system is conserved in time, see Section 2 of [3], for each $\epsilon \in(0,1]$ we have

$$
\left\|\hat{\psi}^{(\epsilon)}(t)\right\|_{L^{2}(\mathbb{T})}=\left\|\hat{\psi}^{(\epsilon)}\right\|_{L^{2}(\mathbb{T})}, \quad t \geq 0, \quad \mathbb{P}_{\epsilon} \text { a.s. }
$$

\subsection{Wigner Functions Corresponding to $\psi^{(\epsilon)}(t)$}

Denote by

$$
\mathfrak{W}_{\epsilon}^{T}(t):=\left[W_{\epsilon,+}(t), Y_{\epsilon,+}(t), Y_{\epsilon,-}(t), W_{\epsilon,-}(t)\right]
$$

the vector made of Wigner functions corresponding to the wave functions $\psi^{(\epsilon)}(t)$. They can be defined by formulas (5.6) and (5.7), where the respective FourierWigner functions $\widehat{W}_{\epsilon, \pm}(t, \eta, k)$ and $\widehat{Y}_{\epsilon, \pm}(t, \eta, k)$ are given by analogues of (5.8) in which the wave functions are substituted by $\psi^{(\epsilon)}(t)$ and the average $\langle\cdot\rangle_{\mu_{\epsilon}}$ is replaced by $\mathbb{E}_{\epsilon}$,

From (5.23) we conclude, thanks to (5.9), that

$$
\sup _{t \geq 0} \sum_{l= \pm}\left(\sup _{\epsilon \in(0,1]}\left\|W_{\epsilon, l}(t)\right\|_{\mathcal{A}^{\prime}}+\sup _{\epsilon \in(0,1]}\left\|Y_{\epsilon, l}(t)\right\|_{\mathcal{A}^{\prime}}\right) \leq 4 K_{0},
$$

where $K_{0}$ is the constant appearing in condition (2.11). As a direct consequence of the above estimate we infer that the components of $\left(\mathfrak{W}_{\epsilon}(\cdot)\right)_{\epsilon \in(0,1]}$ are $*$-weakly sequentially compact in $\left(L^{1}([0,+\infty) ; \mathcal{A})\right)^{*}$ as $\epsilon \rightarrow 0+$, that is given a component of the above family, for example $W_{\epsilon,+}(\cdot)$, and any sequence $\epsilon_{n} \rightarrow 0+$, one can choose a subsequence $W_{\epsilon_{n^{\prime}},+}(\cdot)$ converging $*$-weakly.

To characterize the limit we recall that the thermal energy density $e_{\mathrm{th}}(t, y)$ is given by the solution of the Cauchy problem (3.7), while the mechanical one $e_{\text {mech }}(t, y)$ is defined by (3.4). The limit of the Wigner functions corresponding to the macroscopic profile wave function

$$
\phi(t, y):=\sqrt{\alpha} \kappa(t, y)+i p(t, y), \quad(t, y) \in[0,+\infty) \times \mathbb{R}
$$


equals

$$
\overline{\mathfrak{W}}^{T}(t):=\left[\bar{W}_{+}(t), \bar{Y}_{+}(t), \bar{Y}_{-}(t), \bar{W}_{+}(t)\right]
$$

where

$$
\bar{W}_{ \pm}(t, \mathrm{~d} y, \mathrm{~d} k)=\frac{1}{2}|\phi(t, y)|^{2} \mathrm{~d} y \delta_{0}(\mathrm{~d} k)=e_{\operatorname{mech}}(t, y) \mathrm{d} y \delta_{0}(\mathrm{~d} k),
$$

and $\bar{Y}_{-}(t)=\bar{Y}_{+}^{*}(t)$, with

$$
\bar{Y}_{+}(t, \mathrm{~d} y, \mathrm{~d} k)=\frac{1}{2} \phi^{2}(t, y) \mathrm{d} y \delta_{0}(\mathrm{~d} k)
$$

Our main result concerning the limit of the Wigner transform can be stated as follows.

Theorem 5.1. Suppose that the initial data satisfy the assumptions. Then, $\left(\mathfrak{W}_{\epsilon}(t)\right)_{t \geq 0}$ converge, as $\epsilon \rightarrow 0+, *$-weakly over $\left(L^{1}([0,+\infty), \mathcal{A})\right)^{*}$ to

$$
\mathfrak{W}^{T}(t)=\left[W(t), \bar{Y}_{+}(t), \bar{Y}_{-}(t), W(t)\right], \quad t \geq 0,
$$

where $W(t)$ is a measure on $\mathbb{R} \times \mathbb{T}$ given by

$$
W(t, \mathrm{~d} y, \mathrm{~d} k):=e_{\mathrm{th}}(t, y) \mathrm{d} y \mathrm{~d} k+e_{\operatorname{mech}}(t, y) \mathrm{d} y \delta(\mathrm{d} k) .
$$

Analogously to formulas (5.10) and (5.11), we can write

$$
\epsilon \sum_{x} \mathbb{E}_{\epsilon} \mathfrak{e}_{x}\left(\frac{t}{\epsilon^{2}}\right) J^{*}(\epsilon x)=\left\langle W_{\epsilon, \pm}(t), J\right\rangle
$$

and

$$
\epsilon \sum_{x} \mathbb{E}_{\epsilon}\left[\mathfrak{l}_{x}\left(\frac{t}{\epsilon^{2}}\right) \pm i \sqrt{\alpha} \mathfrak{j}_{x}\left(\frac{t}{\epsilon^{2}}\right)\right] J^{*}(\epsilon x)=\left\langle Y_{\epsilon, \pm}(t), J\right\rangle, \quad J \in \mathcal{S}(\mathbb{R}),
$$

(see (5.12)). Therefore, the conclusion of Theorem 3.2 is a direct consequence of Theorem 5.1.

\section{Evolution of the Wigner Functions}

Using (5.20) we can derive the equations describing the time evolution of the Wigner functions. In particular, one can conclude that for a fixed $\epsilon$ the components of $\left(\mathfrak{W}_{\epsilon}(t)\right)_{t \geq 0}$ belong to $C\left([0,+\infty) ; \mathcal{A}^{\prime}\right)$. After a straightforward calculation (see Section 8 of [8] for details) we obtain that their Fourier transforms satisfy

$$
\partial_{t} \widehat{W}_{\epsilon,+}(t)=-\frac{i}{\epsilon} \delta_{\epsilon} \omega \widehat{W}_{\epsilon,+}(t)+\frac{\gamma}{\epsilon^{2}} \mathcal{L}_{\epsilon \eta} \widehat{W}_{\epsilon,+}(t)-\frac{\gamma}{2 \epsilon^{2}} \sum_{\iota= \pm} \mathcal{L}_{\iota \epsilon \eta}^{+} \widehat{Y}_{\epsilon,-\iota}(t),
$$


and

$$
\begin{aligned}
\partial_{t} \widehat{Y}_{\epsilon,+}(t)= & -\frac{2 i}{\epsilon^{2}} \bar{\omega}_{\widehat{Y}_{\epsilon,+}}(t)+\frac{\gamma}{\epsilon^{2}} \mathcal{L}_{\epsilon \eta} \widehat{Y}_{\epsilon,+}(t) \\
& +\frac{\gamma}{\epsilon^{2}} \mathcal{R}_{\epsilon \eta}\left(\widehat{Y}_{\epsilon,-}-\widehat{Y}_{\epsilon,+}\right)(t)-\frac{\gamma}{2 \epsilon^{2}} \sum_{\iota= \pm} \mathcal{L}_{\iota \epsilon \eta}^{+} \widehat{W}_{\epsilon,-\iota}(t) .
\end{aligned}
$$

Here (cf (5.20))

$$
\begin{aligned}
& \delta_{\epsilon} \omega:=\frac{1}{\epsilon}\left[\omega\left(k+\frac{\epsilon \eta}{2}\right)-\omega\left(k-\frac{\epsilon \eta}{2}\right)\right]=2 \sqrt{\alpha} \mathfrak{s}(\epsilon \eta) \mathfrak{s}(2 k), \\
& \bar{\omega}:=\frac{1}{2}\left[\omega\left(k+\frac{\epsilon \eta}{2}\right)+\omega\left(k-\frac{\epsilon \eta}{2}\right)\right]=4 \sqrt{\alpha}\left[\mathfrak{s}^{2}(k) \mathfrak{c}^{2}\left(\frac{\epsilon \eta}{2}\right)+\mathfrak{c}^{2}(k) \mathfrak{s}^{2}\left(\frac{\epsilon \eta}{2}\right)\right], \\
& \mathcal{L}_{\eta} f(k):=2 \mathcal{R}_{\eta} f(k)-2 f(k) \int_{\mathbb{T}} R\left(k, k^{\prime}, \eta\right) \mathrm{d} k^{\prime} \\
& \mathcal{L}_{\eta}^{ \pm} f(k):=2 \mathcal{R}_{\eta} f(k)-2 R\left(k \pm \frac{\eta}{2}\right) f(k), \\
& \mathcal{R}_{\eta} f(k):=\int_{\mathbb{T}} R\left(k, k^{\prime}, \eta\right) f\left(k^{\prime}\right) \mathrm{d} k^{\prime}
\end{aligned}
$$

where, with some abuse of notation cf (5.21), we have denoted the scattering kernel

$$
R\left(k, k^{\prime}, \eta\right):=\frac{1}{2} \sum_{\iota= \pm 1} r\left(k-\frac{\eta}{2}, k-\iota k^{\prime}\right) r\left(k+\frac{\eta}{2}, k-\iota k^{\prime}\right), \quad k, k^{\prime} \in \mathbb{T} .
$$

A direct calculation yields

$$
R\left(k, k^{\prime}, \eta\right)=R\left(k, k^{\prime}\right)-\mathfrak{s}^{2}\left(\frac{\eta}{2}\right) R_{1}\left(k, k^{\prime}\right)+\mathfrak{s}^{4}\left(\frac{\eta}{2}\right) R_{2}\left(k, k^{\prime} ; \eta\right) .
$$

Here

$$
\begin{aligned}
R\left(k, k^{\prime}\right):=R\left(k, k^{\prime}, 0\right)=\frac{3}{4}\left(\mathfrak{e}_{-} \otimes \mathfrak{e}_{+}+\mathfrak{e}_{+} \otimes \mathfrak{e}_{-}\right)\left(k, k^{\prime}\right), \\
R_{1}\left(k, k^{\prime}\right):=\left(16 \mathfrak{f}_{+} \otimes \mathfrak{f}_{+}+\mathfrak{f}_{+} \otimes \mathfrak{e}_{-}+\mathfrak{e}_{-} \otimes \mathfrak{f}_{+}+3 \mathfrak{f}_{-} \otimes \mathfrak{e}_{+}+3 \mathfrak{e}_{+} \otimes \mathfrak{f}_{-}\right)\left(k, k^{\prime}\right), \\
R_{2}\left(k, k^{\prime} ; \eta\right)=16\left(\mathfrak{f}_{+}(k)+\mathfrak{f}_{+}\left(k^{\prime}\right)\right)+4\left(4 \mathfrak{f}_{+} \otimes \mathfrak{f}_{+}+\mathfrak{f}_{+} \otimes \mathfrak{f}_{-}+\mathfrak{f}_{-} \otimes \mathfrak{f}_{+}\right)\left(k, k^{\prime}\right) \\
\quad-32 \mathfrak{s}^{2}\left(\frac{\eta}{2}\right)\left(\mathfrak{f}_{+}(k)+\mathfrak{f}_{+}\left(k^{\prime}\right)+2 \mathfrak{f}(k)\right)+64 \mathfrak{s}^{4}\left(\frac{\eta}{2}\right) \mathfrak{f}(k),
\end{aligned}
$$

where $\mathfrak{e}_{ \pm}$and $\mathfrak{f}_{ \pm}$are the $L^{1}(\mathbb{T})$ normalized vectors given by

$$
\mathfrak{e}_{+}(k):=\frac{8}{3} \mathfrak{s}^{4}(k), \quad \mathfrak{e}_{-}(k):=2 \mathfrak{s}^{2}(2 k)
$$

and $\mathfrak{f} \equiv 1$,

$$
\mathfrak{f}_{+}(k):=2 \mathfrak{s}^{2}(k), \quad \mathfrak{f}_{-}(k):=2 \mathfrak{c}^{2}(k), \quad k \in \mathbb{T} .
$$


Note also that (cf (5.21))

$$
R(k)=\int_{\mathbb{T}} R\left(k, k^{\prime}\right) \mathrm{d} k^{\prime}=\frac{3}{4} \sum_{\iota \in\{-,+\}} \mathfrak{e}_{\iota}(k) .
$$

In addition,

$$
R^{\prime}(k)=2 \pi(\mathfrak{s}(2 k)+\mathfrak{s}(4 k))
$$

and

$$
R^{\prime \prime}(k)=4 \pi^{2}\left(4 \mathfrak{c}^{2}(2 k)+\mathfrak{c}(2 k)-2\right) .
$$

\subsection{System of Equations for the Laplace-Fourier Transform of the Wigner}

\section{Functions}

Taking the Laplace transform of both sides of (6.1) and (6.2), we get the following equations

$$
\tilde{D}_{1}^{(\epsilon)} w_{\epsilon,+}+\tilde{D}_{+}^{(\epsilon)} y_{\epsilon,+}+\tilde{D}_{-}^{(\epsilon)} y_{\epsilon,-}=\mathcal{R}_{1}^{(\epsilon)}
$$

and

$$
\tilde{D}_{+}^{(\epsilon)} w_{\epsilon,+}+\tilde{D}_{2}^{(\epsilon)} y_{\epsilon,+}+\tilde{D}_{-}^{(\epsilon)} w_{\epsilon,-}=\mathcal{R}_{2}^{(\epsilon)}
$$

Here

$$
\begin{aligned}
w_{\epsilon, \pm}(\lambda, \eta, k) & :=\int_{0}^{+\infty} e^{-\lambda t} \widehat{W}_{\epsilon, \pm}(t, \eta, k) \mathrm{d} t \\
y_{\epsilon, \pm}(\lambda, \eta, k): & =\int_{0}^{+\infty} e^{-\lambda t} \widehat{Y}_{\epsilon, \pm}(t, \eta, k) \mathrm{d} t .
\end{aligned}
$$

In addition, we let

$$
\begin{aligned}
& \tilde{D}_{1}^{(\epsilon)}(\lambda, \eta, k):=\epsilon^{2} \lambda+2 \gamma R_{\epsilon}+i \epsilon \delta_{\epsilon} \omega \\
& \tilde{D}_{2}^{(\epsilon)}(\lambda, \eta, k):=\epsilon^{2} \lambda+2 \gamma R_{\epsilon}+2 i \bar{\omega}, \\
& \tilde{D}_{ \pm}^{(\epsilon)}(\lambda, \eta, k):=-\gamma R_{\epsilon} \pm \frac{\gamma \epsilon}{2} R^{\prime} \eta
\end{aligned}
$$

where

$$
R_{\epsilon}(\eta, k):=R(k)+\frac{(\epsilon \eta)^{2}}{8} R^{\prime \prime}(k) .
$$

Remark. Note that $R^{\prime \prime}(0)>0$. Therefore, given $M>0$ there exists $\epsilon_{0}$ depending only on $M>0$ and such that $R_{\epsilon}(\eta, k)>0$ for all $|\eta| \leq M$ and $k \in \mathbb{T}$.

The right hand sides of (6.11) and (6.12) are respectively equal

$$
\begin{aligned}
& \mathcal{R}_{1}^{(\epsilon)}:=g_{\epsilon}+\epsilon^{2} \widehat{W}_{\epsilon,+}(\eta, k)-\frac{\gamma(\pi \epsilon \eta)^{2}}{2} f_{\epsilon}+\epsilon^{3} r_{\epsilon}^{(1)}, \\
& \mathcal{R}_{2}^{(\epsilon)}:=-g_{\epsilon}+\epsilon^{2} \widehat{Y}_{\epsilon,+}(\eta, k)+\frac{\gamma(\pi \epsilon \eta)^{2}}{2} f_{\epsilon}+\epsilon^{3} r_{\epsilon}^{(2)},
\end{aligned}
$$


where

$$
\begin{aligned}
g_{\epsilon}:= & \frac{3}{2} \gamma \sum_{\iota= \pm} \mathfrak{e}_{\iota}\left\langle v_{\epsilon}, \mathfrak{e}_{-\iota}\right\rangle_{L^{2}(\mathbb{T})}, \\
f_{\epsilon}:= & \mathfrak{f}_{+}\left\langle v_{\epsilon}, 16 \mathfrak{f}_{+}+\mathfrak{e}_{-}\right\rangle_{L^{2}(\mathbb{T})}+\mathfrak{e}_{-}\left\langle v_{\epsilon}, \mathfrak{f}_{+}\right\rangle_{L^{2}(\mathbb{T})} \\
& +3 \mathfrak{f}_{-}\left\langle v_{\epsilon}, \mathfrak{e}_{+}\right\rangle_{L^{2}(\mathbb{T})}+3 \mathfrak{e}_{+}\left\langle v_{\epsilon}, \mathfrak{f}_{-}\right\rangle_{L^{2}(\mathbb{T})} .
\end{aligned}
$$

Here, for the abbreviation sake we have let

$$
v_{\epsilon}(\lambda, \eta, k):=w_{\epsilon,+}(\lambda, \eta, k)-\frac{1}{2} y_{\epsilon, o}(\lambda, \eta, k),
$$

where

$$
y_{\epsilon, o}(\lambda, \eta, k):=y_{\epsilon,+}(\lambda, \eta, k)+y_{\epsilon,-}(\lambda, \eta, k) .
$$

In addition, the remainder terms $r_{\epsilon}^{(i)}, i=1,2$ satisfy

$$
\limsup _{\epsilon \rightarrow 0+} \sup _{\lambda \geq \lambda_{0}}\left\|r_{\epsilon}^{(i)}(\lambda)\right\|_{\mathcal{A}_{M}^{\prime}}<+\infty, \quad i=1,2, \lambda_{0}, M>0 .
$$

A closed system of equations on

$$
\mathfrak{w}_{\epsilon}^{T}(\lambda, \eta, k):=\left[w_{\epsilon,+}, y_{\epsilon,+}, y_{\epsilon,-}, w_{\epsilon,-}\right]
$$

can be rewritten in the matrix form

$$
\tilde{D}_{\epsilon} \mathfrak{w}_{\epsilon}=\mathfrak{R}_{\epsilon},
$$

where

$$
\mathfrak{R}_{\epsilon}^{T}:=\left[\mathcal{R}_{1}^{(\epsilon)}, \mathcal{R}_{2}^{(\epsilon)}, \mathcal{R}_{2,-}^{(\epsilon)}, \mathcal{R}_{1,-}^{(\epsilon)}\right]
$$

and $\tilde{D}_{\epsilon}$ is a $4 \times 4$ matrix that can be written in the block form

$$
\tilde{D}_{\epsilon}=\left[\begin{array}{cc}
A_{\epsilon} & B_{\epsilon} \\
B_{\epsilon} & C_{\epsilon}
\end{array}\right],
$$

where $A_{\epsilon}, B_{\epsilon}, C_{\epsilon}$ are $2 \times 2$ matrices given by

$$
A_{\epsilon}:=\left[\begin{array}{cc}
\tilde{D}_{1}^{(\epsilon)} & \tilde{D}_{+}^{(\epsilon)} \\
\tilde{D}_{+}^{(\epsilon)} & \tilde{D}_{2}^{(\epsilon)}
\end{array}\right], \quad C_{\epsilon}:=\left[\begin{array}{cc}
\left(\tilde{D}_{2}^{(\epsilon)}\right)^{*} & \tilde{D}_{+}^{(\epsilon)} \\
\tilde{D}_{+}^{(\epsilon)} & \left(\tilde{D}_{1}^{(\epsilon)}\right)^{*}
\end{array}\right]
$$

and $B_{\epsilon}=\tilde{D}_{-}^{(\epsilon)} I_{2}$, with $I_{n}$ denoting the $n \times n$ identity matrix.

We have also denoted

$$
\mathcal{R}_{1,-}^{(\epsilon)}(\lambda, \eta, k):=\mathcal{R}_{1}^{(\epsilon)}(\lambda, \eta,-k), \quad \mathcal{R}_{2,-}^{(\epsilon)}(\lambda, \eta, k):=\left(\mathcal{R}_{2}^{(\epsilon)}(\lambda,-\eta, k)\right)^{*} .
$$


Let $\mathfrak{w}_{\epsilon}^{(\iota)}(\lambda, \eta)$ be the column vectors obtained by scalar multiplication of each component of $\mathfrak{w}_{\epsilon}(\lambda, \eta, k)$ by $\mathfrak{e}_{\iota}$. Note that

$$
\mathfrak{R}_{\epsilon}=\frac{3 \gamma}{2} \sum_{\iota= \pm} \mathfrak{e}_{\iota} F \mathfrak{w}_{\epsilon}^{(-\iota)}(\lambda, \eta)+\epsilon^{2} \mathfrak{h}_{\epsilon},
$$

where the matrix $F=(1 / 2) \mathrm{e}^{T} \otimes \mathrm{e}$, vector $\mathrm{e}^{T}:=[1,-1,-1,1]$,

$$
\mathfrak{h}_{\epsilon}(\lambda, \eta, k)=\widehat{\mathfrak{W}}_{\epsilon}(\eta, k)-\frac{\gamma(\pi \eta)^{2}}{2} f_{\epsilon} \mathrm{e}+\mathfrak{r}_{\epsilon}(\lambda, \eta, k)
$$

and $\widehat{\mathfrak{W}}_{\epsilon}(\eta, k)$ is the column vector corresponding to the Fourier-Wigner transforms of the components of (5.5) and

$$
\begin{gathered}
\mathfrak{r}_{\epsilon}^{T}:=\left[r_{\epsilon}^{(1)}, r_{\epsilon}^{(2)}, r_{\epsilon,-}^{(2)}, r_{\epsilon,-}^{(1)}\right], \\
r_{\epsilon,-}^{(1)}(\lambda, \eta, k):=r_{\epsilon}^{(1)}(\lambda, \eta,-k), \quad r_{\epsilon,-}^{(2)}(\lambda, \eta, k):=\left(r_{\epsilon}^{(2)}(\lambda,-\eta, k)\right)^{*} .
\end{gathered}
$$

Recall that $\mathrm{a} \otimes \mathrm{b}=\left[a_{i} b_{j}\right]$, if $\mathrm{a}=\left[a_{1}, \ldots, a_{n}\right]$ and $\mathrm{b}=\left[b_{1}, \ldots, b_{m}\right]$.

\subsection{Invertibility of Matrix $\tilde{D}_{\epsilon}$}

We prove that the matrix $\tilde{D}_{\epsilon}$ appearing in (6.20) is invertible, thus the vector of the Laplace-Fourier transforms of Wigner functions is uniquely determined by the system. It turns out to be true, provided that $\lambda$ is sufficiently large.

Let us denote $\tilde{\delta}_{\epsilon}(\lambda, \eta, k):=\operatorname{det} \tilde{D}_{\epsilon}(\lambda, \eta, k)$. Since matrices $B_{\epsilon}$ and $C_{\epsilon}$ commute we have (see p. 56 of [7])

$$
\begin{aligned}
\tilde{\delta}_{\epsilon} & =\operatorname{det}\left(A_{\epsilon} C_{\epsilon}-B_{\epsilon}^{2}\right) \\
& =\left|\tilde{D}_{1}^{(\epsilon)}\left(\tilde{D}_{2}^{(\epsilon)}\right)^{*}+\left[\tilde{D}_{+}^{(\epsilon)}\right]^{2}-\left[\tilde{D}_{-}^{(\epsilon)}\right]^{2}\right|^{2}-4\left[\tilde{D}_{+}^{(\epsilon)}\right]^{2} \operatorname{Re} \tilde{D}_{1}^{(\epsilon)} \operatorname{Re} \tilde{D}_{2}^{(\epsilon)}
\end{aligned}
$$

After a direct calculation we get

$$
\begin{aligned}
\tilde{\delta}_{\epsilon}= & \epsilon^{8} \lambda^{4}+8 \epsilon^{6} \lambda^{3} \gamma R_{\epsilon}+4 \epsilon^{4} \lambda^{2}\left[5\left(\gamma R_{\epsilon}\right)^{2}+\bar{\omega}^{2}+\left(\frac{\epsilon \delta_{\epsilon} \omega}{2}\right)^{2}-\left(\frac{\gamma \epsilon R^{\prime} \eta}{2}\right)^{2}\right] \\
& +4 \epsilon^{2} \lambda\left(\gamma R_{\epsilon}\right)\left[4\left(\gamma R_{\epsilon}\right)^{2}+4 \bar{\omega}^{2}+\left(\epsilon \delta_{\epsilon} \omega\right)^{2}-\left(\gamma \epsilon R^{\prime} \eta\right)^{2}\right] \\
& +4 \epsilon^{2}\left[\left(\gamma R_{\epsilon} \delta_{\epsilon} \omega\right)^{2}-2\left(\gamma R_{\epsilon}\right) \delta_{\epsilon} \omega \bar{\omega} \gamma R^{\prime} \eta+\left(\bar{\omega} \delta_{\epsilon} \omega\right)^{2}\right]+16\left(\gamma R_{\epsilon} \bar{\omega}\right)^{2} .
\end{aligned}
$$

Define

$$
\tilde{\delta}_{\epsilon}^{(0)}:=\left(\epsilon^{2} \lambda+R_{\epsilon}\right)^{4} .
$$

In what follows, we shall assume that given $M>0$ one considers only $\epsilon \epsilon$ $\left(0, \epsilon_{0}(M)\right]$ such that $R_{\epsilon}(\eta, k)>0$ for all $|\eta| \leq M$ and $k \in \mathbb{T}$, see the Remark after formula (6.14). 
Proposition 6.1. For any $M>0$ there exist $\epsilon_{0}(M), \lambda_{0}(M)>0$ such that

$$
\tilde{\delta}_{\epsilon}(\lambda, \eta, k) \asymp \tilde{\delta}_{\epsilon}^{(0)}(\lambda, \eta, k), \quad k \in \mathbb{T}, \quad|\eta| \leq M, \lambda>\lambda_{0}, \quad \epsilon \in\left(0, \epsilon_{0}\right] .
$$

In particular, we have

$$
\tilde{\delta}_{\epsilon}(\lambda, \eta, k)>0, \quad k \in \mathbb{T},|\eta| \leq M, \lambda>\lambda_{0}, \quad \epsilon \in\left(0, \epsilon_{0}\right] .
$$

Proof. Using (6.10) we conclude that for any $M>0$ there is $\epsilon_{0}>0$ such that

$$
R_{\epsilon} \asymp R(k)+(\epsilon \eta)^{2}, \quad k \in \mathbb{T},|\eta| \leq M, \quad \epsilon \in\left(0, \epsilon_{0}\right] .
$$

Comparing the second formula from (6.3) with (6.30) we get

$$
\bar{\omega} \asymp R_{\epsilon}, \quad k \in \mathbb{T}, \quad|\eta| \leq M, \quad \epsilon \in\left(0, \epsilon_{0}\right] .
$$

From (6.9), the first formula of (6.3) and (6.31) we get also

$$
\left|\delta_{\epsilon} \omega R^{\prime}\right| \preceq R_{\epsilon}, \quad|\eta| \leq M, \quad k \in \mathbb{T} .
$$

Therefore

$$
8(\epsilon \gamma)^{2} R_{\epsilon}\left|\delta_{\epsilon} \omega \bar{\omega} R^{\prime} \eta\right| \preceq \epsilon^{2} R_{\epsilon}^{3}, \quad|\eta| \leq M, \quad k \in \mathbb{T}, \quad \epsilon \in\left(0, \epsilon_{0}\right] .
$$

Choosing $\lambda_{0}$ sufficiently large we can guarantee also that

$$
\epsilon^{2} \lambda\left(\gamma R_{\epsilon}\right)^{3} \geq 8(\epsilon \gamma)^{2} R_{\epsilon}\left|\delta_{\epsilon} \omega \bar{\omega} R^{\prime} \eta\right|
$$

for $|\eta| \leq M, k \in \mathbb{T}, \lambda>\lambda_{0}, \epsilon \in\left(0, \epsilon_{0}\right]$.

In a similar fashion we can argue that

$$
\epsilon^{4} \lambda^{2}\left(\gamma R_{\epsilon}\right)^{2} \geq 4 \epsilon^{4} \lambda\left(\gamma R_{\epsilon}\right)\left[\left(\delta_{\epsilon} \omega\right)^{2}-\left(\gamma \hat{R}^{\prime} \eta\right)^{2}\right]
$$

and

$$
\epsilon^{6} \lambda^{3} \gamma R_{\epsilon} \geq \epsilon^{6} \lambda^{2}\left[\left(\delta_{\epsilon} \omega\right)^{2}-\left(\gamma R^{\prime} \eta\right)^{2}\right]
$$

for $|\eta| \leq M, k \in \mathbb{T}, \lambda>\lambda_{0}, \epsilon \in\left(0, \epsilon_{0}\right]$. From estimates (6.33)-(6.35) we conclude that

$$
\tilde{\delta}_{\epsilon} \succeq \epsilon^{8} \lambda^{4}+\epsilon^{6} \lambda^{3} R_{\epsilon}+\epsilon^{4} \lambda^{2} R_{\epsilon}^{2}+\epsilon^{2} \lambda R_{\epsilon}^{3}+R_{\epsilon}^{4}
$$

Therefore, cf (6.27), we get $\tilde{\delta}_{\epsilon}^{(0)} \preceq \tilde{\delta}_{\epsilon}$. The reverse estimate is a simple consequence of the first two formulas from (6.3) and (6.32). 


\subsection{Inverse of $\tilde{D}_{\epsilon}(\lambda, p, k)$}

Recall that $\tilde{D}_{\epsilon}(\lambda, p, k)$ is a $2 \times 2$ block matrix of the form (6.21). Since $B_{\epsilon}$ is diagonal we have $\left[A_{\epsilon}, B_{\epsilon}\right]=\left[C_{\epsilon}, B_{\epsilon}\right]=0$. A simple calculation shows that also

$$
A_{\epsilon} C_{\epsilon}=C_{\epsilon} A_{\epsilon} .
$$

Therefore,

$$
\tilde{D}_{\epsilon}^{-1}=\left[\begin{array}{cc}
\left(C_{\epsilon} A_{\epsilon}-B_{\epsilon}^{2}\right)^{-1} & 0 \\
0 & \left(C_{\epsilon} A_{\epsilon}-B_{\epsilon}^{2}\right)^{-1}
\end{array}\right]\left[\begin{array}{cc}
C_{\epsilon} & -B_{\epsilon} \\
-B_{\epsilon} & A_{\epsilon}
\end{array}\right],
$$

provided that $\operatorname{det} \tilde{D}_{\epsilon} \neq 0$. Note that

$$
\begin{aligned}
& \left(C_{\epsilon} A_{\epsilon}-B_{\epsilon}^{2}\right)^{-1}
\end{aligned}
$$

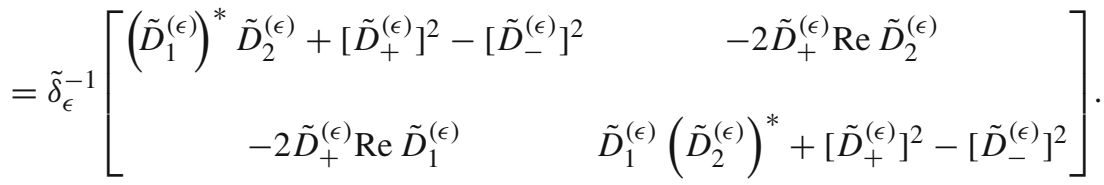

Substituting into (6.37), using also (6.22) we conclude that the inverse matrix $\tilde{D}_{\epsilon}^{-1}$ is a $2 \times 2$ block matrix of the form $\tilde{D}_{\epsilon}^{-1}=\tilde{\delta}_{\epsilon}^{-1} \operatorname{adj}\left(\tilde{D}_{\epsilon}\right)$ where the adjugate of $\tilde{D}_{\epsilon}$ equals

$$
\operatorname{adj}\left(\tilde{D}_{\epsilon}\right)=\left[\begin{array}{ll}
P_{\epsilon} & Q_{\epsilon} \\
Q_{\epsilon} & M_{\epsilon}
\end{array}\right]
$$

where $M_{\epsilon}, P_{\epsilon}$ and $Q_{\epsilon}$ are $2 \times 2$ matrices given by

$$
\begin{aligned}
P_{\epsilon} & :=\left[\begin{array}{ll}
\tilde{d}_{1}^{(\epsilon)} & \tilde{d}_{-}^{(\epsilon)} \\
\tilde{d}_{-}^{(\epsilon)} & \tilde{d}_{2}^{(\epsilon)}
\end{array}\right], \quad Q_{\epsilon}:=\left[\begin{array}{ll}
\left(\tilde{d}_{+}^{(\epsilon)}\right)^{*} & \tilde{d}_{o}^{(\epsilon)} \\
\tilde{d}_{o}^{(\epsilon)} & \tilde{d}_{+}^{(\epsilon)}
\end{array}\right], \\
M_{\epsilon} & :=\left[\begin{array}{ll}
\left(\tilde{d}_{2}^{(\epsilon)}\right)^{*} & \left(\tilde{d}_{-}^{(\epsilon)}\right)^{*} \\
\left(\tilde{d}_{-}^{(\epsilon)}\right)^{*} & \left(\tilde{d}_{1}^{(\epsilon)}\right)^{*}
\end{array}\right] .
\end{aligned}
$$

Here

$$
\begin{aligned}
& \tilde{\mathrm{d}}_{1}^{(\epsilon)}:=\left|\tilde{D}_{2}^{(\epsilon)}\right|^{2}\left(\tilde{D}_{1}^{(\epsilon)}\right)^{*}-\left(\left(\tilde{D}_{+}^{(\epsilon)}\right)^{2}+\left(\tilde{D}_{-}^{(\epsilon)}\right)^{2}\right) \operatorname{Re} \tilde{D}_{2}^{(\epsilon)} \\
& -i\left(\left(\tilde{D}_{+}^{(\epsilon)}\right)^{2}-\left(\tilde{D}_{-}^{(\epsilon)}\right)^{2}\right) \operatorname{Im} \tilde{D}_{2}^{(\epsilon)} \\
& \tilde{\mathrm{d}}_{2}^{(\epsilon)}:=\left|\tilde{D}_{1}^{(\epsilon)}\right|^{2}\left(\tilde{D}_{2}^{(\epsilon)}\right)^{*}-\left(\left(\tilde{D}_{+}^{(\epsilon)}\right)^{2}+\left(\tilde{D}_{-}^{(\epsilon)}\right)^{2}\right) \operatorname{Re} \tilde{D}_{1}^{(\epsilon)} \\
& -i\left(\left(\tilde{D}_{+}^{(\epsilon)}\right)^{2}-\left(\tilde{D}_{-}^{(\epsilon)}\right)^{2}\right) \operatorname{Im} \tilde{D}_{1}^{(\epsilon)}, \\
& \tilde{\mathrm{d}}_{-}^{(\epsilon)}:=\tilde{D}_{+}^{(\epsilon)}\left(\left(\tilde{D}_{+}^{(\epsilon)}\right)^{2}-\left(\tilde{D}_{-}^{(\epsilon)}\right)^{2}\right)-\tilde{D}_{+}^{(\epsilon)}\left(\tilde{D}_{1}^{(\epsilon)} \tilde{D}_{2}^{(\epsilon)}\right)^{*}, \\
& \tilde{\mathrm{d}}_{+}^{(\epsilon)}:=-\tilde{D}_{-}^{(\epsilon)}\left(\tilde{D}_{1}^{(\epsilon)}\left(\tilde{D}_{2}^{(\epsilon)}\right)^{*}+\left(\tilde{D}_{+}^{(\epsilon)}\right)^{2}-\left(\tilde{D}_{-}^{(\epsilon)}\right)^{2}\right), \\
& \tilde{\mathrm{d}}_{o}^{(\epsilon)}:=2 \tilde{D}_{+}^{(\epsilon)} \tilde{D}_{-}^{(\epsilon)} \operatorname{Re} \tilde{D}_{2}^{(\epsilon)} \text {. }
\end{aligned}
$$


For the abbreviation sake we denote by $\mathfrak{d}_{j, \epsilon}, j=1, \ldots, 4$ the vectors corresponding to the rows of the adjugate of $\tilde{D}_{\epsilon}$ given by (6.38). Combining the above with (6.13) and (6.26) we get.

Proposition 6.2. For any $M, \lambda>0$ we have

$$
\begin{aligned}
\tilde{\mathrm{d}}_{1}^{(\epsilon)} & =4(\gamma R)^{3}+8 \gamma R \omega^{2}+o_{\epsilon}(\eta, k), \\
\tilde{\mathrm{d}}_{2}^{(\epsilon)} & =4(\gamma R)^{3}-8 i(\gamma R)^{2} \omega+o_{\epsilon}(\eta, k), \\
\tilde{\mathrm{d}}_{ \pm}^{(\epsilon)} & =4(\gamma R)^{3}-4 i(\gamma R)^{2} \omega+o_{\epsilon}(\eta, k), \\
\tilde{\mathrm{d}}_{o}^{(\epsilon)} & =4(\gamma R)^{3}+o_{\epsilon}(\eta, k), \\
\tilde{\delta}_{\epsilon} & =16(\gamma R \omega)^{2}+o_{\epsilon}(\eta, k)
\end{aligned}
$$

where $\lim _{\epsilon \rightarrow 0+} \sup _{|\eta| \leq M} o_{\epsilon}(\eta, k)=0$ for any $k \in \mathbb{T}$.

\section{Proof of Theorem 5.1}

As we have already mentioned, for any sequence $\epsilon_{n} \rightarrow 0+$ there exists a subsequence $\left(\mathfrak{W}_{\epsilon_{n^{\prime}}}(t)\right)$ that convergences $*$-weakly to some $\mathfrak{W} \in\left(L^{1}([0,+\infty) ; \mathcal{A})\right)^{*}$. We prove that the element $\mathfrak{W}$ does not depend on the choice of the sequence $\epsilon_{n^{\prime}}$ by showing that for any $M>0$ there exists $\lambda_{0}>0$ such that the vector $\left(\mathfrak{w}_{\epsilon_{n^{\prime}}}(\lambda)\right)$ made of Laplace transforms of the components of $\mathfrak{W}_{\epsilon_{n^{\prime}}}(t)$ converges $*$-weakly over $\mathcal{A}_{M}^{\prime}$ for any $\lambda>\lambda_{0}$. In fact one can describe the respective limit as the Laplace transform of the vector $\mathfrak{W}(t)$ appearing in the statement of Theorem 5.1. This identifies the limit of $\left(\mathfrak{W}_{\epsilon}(t)\right)$, as $\epsilon \rightarrow 0+$ finishing in this way the proof of Theorem 5.1.

From (6.20) we obtain

$$
\mathfrak{w}_{\epsilon}=\tilde{D}_{\epsilon}^{-1} \mathfrak{R}_{\epsilon} \text {. }
$$

Unfortunately, the right hand side of the above system contains also terms that depend on the vector $\mathfrak{w}_{\epsilon}$, via the projections of its components onto the vectors $\mathfrak{e}_{ \pm}$ and $\mathfrak{f}_{ \pm}$. To describe the behavior of $\mathfrak{w}_{\epsilon}$ we need to determine first these projections.

Using (6.20) the above system can be rewritten in the form

$$
\frac{1}{\epsilon^{2}}\left(\mathfrak{w}_{\epsilon}-\frac{3 \gamma}{2} \sum_{\iota= \pm} \mathfrak{e}_{\iota} \tilde{E}_{\epsilon} \mathfrak{w}_{\epsilon}^{(-\iota)}\right)=\mathfrak{z} \epsilon,
$$

where

$$
\mathfrak{z}_{\epsilon}^{T}(\lambda, \eta, k)=\left[z_{\epsilon}^{(1)}, z_{\epsilon}^{(2)}, z_{\epsilon,-}^{(2)}, z_{\epsilon,-}^{(1)}\right]:=\tilde{D}_{\epsilon}^{-1} \mathfrak{h}_{\epsilon},
$$

$\mathfrak{h}_{\epsilon}$ is given by (6.25) and the $4 \times 4$ matrix $\tilde{E}_{\epsilon}(\lambda, \eta, k)$ equals

$$
\tilde{E}_{\epsilon}:=\frac{1}{2} \mathrm{e}^{T} \otimes \Delta_{\epsilon}
$$

with $\Delta_{\epsilon}^{T}:=\left[\tilde{\Delta}_{1, \epsilon}, \tilde{\Delta}_{2, \epsilon}, \tilde{\Delta}_{2, \epsilon}^{*}, \tilde{\Delta}_{1, \epsilon}^{*}\right]$ and

$$
\begin{aligned}
& \tilde{\Delta}_{1, \epsilon}:=\tilde{\mathrm{d}}_{1}^{(\epsilon)}+\tilde{\mathrm{d}}_{o}^{(\epsilon)}-\tilde{\mathrm{d}}_{-}^{(\epsilon)}-\left(\tilde{\mathrm{d}}_{+}^{(\epsilon)}\right)^{*}, \\
& \tilde{\Delta}_{2, \epsilon}:=\tilde{\mathrm{d}}_{-}^{(\epsilon)}+\tilde{\mathrm{d}}_{+}^{(\epsilon)}-\tilde{\mathrm{d}}_{2}^{(\epsilon)}-\tilde{\mathrm{d}}_{o}^{(\epsilon)} .
\end{aligned}
$$


Multiplying both sides of (7.2) by $\mathfrak{e}_{\iota}, \iota \in\{-,+\}$ and then integrating over $\mathbb{T}$ we get a system of 8 equations

$$
G_{\epsilon} \mathfrak{u}_{\epsilon}=\mathfrak{v}_{\epsilon},
$$

where

$$
\mathfrak{u}_{\epsilon}(\lambda, \eta):=\left[\begin{array}{c}
\mathfrak{w}_{\epsilon}^{(-)} \\
\mathfrak{w}_{\epsilon}^{(+)}
\end{array}\right], \quad \mathfrak{v}_{\epsilon}(\lambda, \eta):=\left[\begin{array}{c}
\mathfrak{z}_{\epsilon}^{(-)} \\
\mathfrak{z}_{\epsilon}^{(+)}
\end{array}\right] .
$$

Here $\mathfrak{w}_{\epsilon}^{(\iota)}$ are column vectors obtained by a scalar multiplication of the entries of $\mathfrak{w}_{\epsilon}($ see $(6.19))$ by $\mathfrak{e}_{\iota}$. The same concerns

$$
\left(\mathfrak{z}_{\epsilon}^{(\iota)}\right)^{T}(\lambda, \eta, k)=\left[z_{\epsilon}^{(1, \iota)}, z_{\epsilon}^{(2, \iota)}, z_{\epsilon,-}^{(2, \iota)}, z_{\epsilon,-}^{(1, \iota)}\right] .
$$

Matrix $G_{\epsilon}(\lambda, \eta)$ is a $2 \times 2$ block matrix of the form

$$
G_{\epsilon}=\left[\begin{array}{cc}
A_{o}^{(\epsilon)} & A_{-}^{(\epsilon)} \\
A_{+}^{(\epsilon)} & A_{o}^{(\epsilon)}
\end{array}\right],
$$

where $A_{o}^{(\epsilon)}, A_{ \pm}^{(\epsilon)}$ are $4 \times 4$ matrices defined as follows:

$$
\begin{aligned}
& A_{\iota}^{(\epsilon)}:=-\frac{3 \gamma}{2 \epsilon^{2}} \int_{\mathbb{T}} \frac{\mathfrak{e}_{l}^{2}}{\tilde{\delta}_{\epsilon}} \tilde{E}_{\epsilon} \mathrm{d} k, \quad \iota \in\{-,+\}, \\
& A_{o}^{(\epsilon)}:=\epsilon^{-2}\left(I-\frac{3 \gamma}{2} \int_{\mathbb{T}} \frac{\mathfrak{e}_{-} \mathfrak{e}_{+}}{\tilde{\delta}_{\epsilon}} \tilde{E}_{\epsilon} \mathrm{d} k\right) .
\end{aligned}
$$

Note that vector $\mathfrak{v}_{\epsilon}$ appearing on the right hand side of (7.6) still depends on the projections of $\mathfrak{w}_{\epsilon}$ onto $\mathfrak{f}_{ \pm}, \mathrm{cf}$ (6.25) and (6.16). It turns out however that the asymptotics of these projections, as $\epsilon \rightarrow 0+$, can be described by only one of them, for example $\mathfrak{w}_{\epsilon}^{(-)}$. This is a conclusion of our next result. Denote by $\delta w_{\epsilon}:=$ $w_{\epsilon}^{(+)}-w_{\epsilon}^{(-)}$. We shall also use the following convention: for a given $M>0$ the constants $\epsilon_{0}, \lambda_{0}>0$ are selected as in the statement of Proposition 6.1 so that $\tilde{\delta}_{\epsilon}(\lambda, \eta, k) \asymp \tilde{\delta}_{\epsilon}^{(0)}(\lambda, \eta, k)$ for all $k \in \mathbb{T},|\eta| \leq M$ and $\lambda>\lambda_{0}$. In particular, then we have (7.1).

Theorem 7.1. For any $M>0$ and $\lambda>\lambda_{0}$ we have (see Section 4 for the definition of $\preceq$ )

$$
\left|\delta w_{\epsilon}(\lambda, \eta)\right| \preceq \epsilon^{2}
$$

and

$$
\left|y_{\epsilon, \iota^{\prime}}^{(\iota)}(\lambda, \eta)\right| \preceq \epsilon^{2}, \quad \epsilon \in\left(0, \epsilon_{0}\right], \quad|\eta| \leq M, \iota, \iota^{\prime} \in\{-,+\} .
$$

Moreover, for any $|\eta| \leq M$ and $\lambda>\lambda_{0}$ we have

$$
\lim _{\epsilon \rightarrow 0+} \int_{\mathbb{T}}\left|w_{\epsilon,+}(\lambda, \eta, k)-w_{\epsilon}^{(-)}(\lambda, \eta)\right| R(k) \mathrm{d} k=0
$$

and

$$
\lim _{\epsilon \rightarrow 0+} \int_{\mathbb{T}}\left|y_{\epsilon, \pm}(\lambda, \eta, k)\right| R(k) \mathrm{d} k=0 .
$$


The proof of the theorem is presented in Section 8.

To describe the limit of $w_{\epsilon}^{(-)}(\lambda, \eta)$ we can use the the system (7.6), which is "almost closed" with respect to the components of $\mathfrak{w}_{\epsilon}^{(-)}$, that is it is closed modulo some corrections that in light of Theorem 7.1 are of lower order of magnitude.

Let us first introduce some additional notation. Given the wave function $\phi(t, y)$ we define the vector of the Laplace-Fourier transforms of the respective macroscopic Wigner functions

$$
\mathfrak{w}_{\phi}^{T}(\lambda, \eta, h)=\left[w_{\phi,+}, y_{\phi,+}, y_{\phi,-}, w_{\phi,-}\right],
$$

where

$$
\begin{aligned}
w_{\phi, \pm}(\lambda, \eta, h) & :=\int_{0}^{+\infty} e^{-\lambda t} \widehat{W}_{\phi,+}(t, \eta, h) \mathrm{d} t, \\
y_{\phi, \pm}(\lambda, \eta, h) & :=\int_{0}^{+\infty} e^{-\lambda t} \widehat{Y}_{\phi,+}(t, \eta, h) \mathrm{d} t, \quad \lambda>0,(\eta, h) \in \mathbb{R}^{2} .
\end{aligned}
$$

Here

$$
\begin{aligned}
\widehat{W}_{\phi,+}(t, \eta, h) & :=\frac{1}{2} \hat{\phi}^{*}\left(t, h-\frac{\eta}{2}\right) \hat{\phi}\left(t, h+\frac{\eta}{2}\right) \\
\widehat{Y}_{\phi,+}(t, \eta, h) & :=\frac{1}{2} \hat{\phi}\left(t,-h+\frac{\eta}{2}\right) \hat{\phi}\left(t, h+\frac{\eta}{2}\right), \\
\widehat{Y}_{\phi,-}(t, \eta, k) & :=\left(\widehat{Y}_{\phi,+}\right)^{*}(t,-\eta, h), \quad \widehat{W}_{\phi,-}(t, \eta, h):=\widehat{W}_{\phi,+}(t, \eta,-h) .
\end{aligned}
$$

Define $\overline{\mathfrak{w}}_{\phi}^{T}(\lambda, \eta):=\left[\bar{w}_{\phi}, \bar{y}_{\phi,+}, \bar{y}_{\phi,-}, \bar{w}_{\phi}\right]$, where

$$
\bar{w}_{\phi}(\lambda, \eta):=\int_{\mathbb{R}} w_{\phi, \pm}(\lambda, \eta, h) \mathrm{d} h, \bar{y}_{\phi, \pm}(\lambda, \eta):=\int_{\mathbb{R}} y_{\phi, \pm}(\lambda, \eta, h) \mathrm{d} h .
$$

Define $w^{(-)}(\lambda, \eta)$ by the formula

$$
\begin{aligned}
& \left(\lambda+\hat{c}(2 \pi \eta)^{2}\right) w^{(-)}(\lambda, \eta)=-3 \gamma(\pi \eta)^{2} \overline{\mathfrak{w}}_{\phi}(\lambda, \eta) \cdot \mathrm{e} \\
& \quad+12 \gamma \pi^{2} \int_{\mathbb{R}} h^{2} \mathfrak{w}_{\phi}(\lambda, \eta, h) \cdot \mathrm{ed} h+\hat{e}_{\mathrm{th}}^{(0)}(\eta), \quad(\lambda, \eta) \in(0,+\infty) \times \mathbb{R} .
\end{aligned}
$$

Here $\hat{e}_{\mathrm{th}}(\eta)$ is the Fourier transform of $e_{\mathrm{th}}(y)$ appearing in (3.5) and $\hat{c}$ is given by (3.8).

We can show, see Section 9 below for the proof, the following result.

Theorem 7.2. For any $M>0$ and $J \in \mathcal{S}(\mathbb{R})$ such that supp $\hat{J} \subset[-M, M]$ we have

$$
\int_{\mathbb{R}} w^{(-)}(\lambda, \eta) \hat{J}^{*}(\eta) \mathrm{d} \eta=\lim _{\epsilon \rightarrow 0+} \int_{\mathbb{R}} w_{\epsilon}^{(-)}(\lambda, \eta) \hat{J}^{*}(\eta) \mathrm{d} \eta
$$

for all $\lambda>\lambda_{0}$. 
To obtain the asymptotics of $\mathfrak{w}_{\epsilon}(\lambda, \eta, k)$ we use (7.1), which allows us to describe the Fourier-Laplace transforms of the Wigner functions in terms of their projections onto $\mathfrak{e}_{ \pm}$and $\mathfrak{f}_{ \pm}$. We obtain then the following result.

Theorem 7.3. For any $M>0$ we have

$$
\lim _{\epsilon \rightarrow 0+}\left|\int_{\mathbb{T}} w_{\epsilon,+}(\lambda, \eta, k) \varphi(k) \mathrm{d} k-w_{\epsilon}^{(-)}(\lambda, \eta) \int_{\mathbb{T}} \varphi(k) \mathrm{d} k-\bar{w}_{\phi}(\lambda, \eta) \varphi(0)\right|=0
$$

and

$$
\lim _{\epsilon \rightarrow 0+} \int_{\mathbb{T}} y_{\epsilon, \pm}(\lambda, \eta, k) \varphi(k) d k=\bar{y}_{\phi, \pm}(\lambda, \eta) \varphi(0),
$$

for all $|\eta| \leq M, \lambda>\lambda_{0}$ and $\varphi \in C(\mathbb{T})$.

The proof of this result is contained in Section 10.

\section{The End of the Proof of Theorem 5.1}

Thanks to (5.24) we know that $\mathfrak{W}_{\epsilon}(t)$ is sequentially pre-compact, as $\epsilon \rightarrow 0+$, in the $*$-weak topology of $\left(L^{1}([0,+\infty), \mathcal{A})\right)^{*}$. To identify its limiting points we consider $\mathfrak{w}_{\epsilon}(\lambda, \eta, k)$ the vector of the Laplace-Fourier transforms of $\mathfrak{W}_{\epsilon}(t)$. Given $\lambda>0$ this family is sequentially pre-compact in the $*$-weak topology of $\mathcal{A}^{\prime}$, as $\epsilon \rightarrow 0+$. Thanks to Theorems 7.2 and 7.3 we conclude that given $M>0$ one can choose $\lambda_{0}$ as in the statement of Proposition 6.1, such that the the components of $\mathfrak{w}_{\epsilon}(\lambda, \eta, k)$ converge $*$-weakly over $\mathcal{A}_{M}^{\prime}$ to the Laplace-Fourier transforms of the respective functions appearing in the claim of Theorem 5.1 for any $\lambda>\lambda_{0}$. To finish the proof we only need to verify that $w(\lambda, \mathrm{d} \eta, \mathrm{d} k)$ - the limit of $w_{\epsilon,+}(\lambda, \eta, k)$ (the limit of $w_{\epsilon,-}$ can then be trivially concluded) agrees for $\lambda>\lambda_{0}$ with the Laplace transform of $W(t, \mathrm{~d} y, \mathrm{~d} k)$ appearing in (5.30).

According to Theorem 7.3 the limit in question is the Fourier-Laplace transform of the measure-valued function

$$
W^{\prime}(t, \mathrm{~d} y, \mathrm{~d} k)=e_{\mathrm{th}}^{\prime}(t, y) \mathrm{d} y \mathrm{~d} k+e_{\operatorname{mech}}(t, y) \mathrm{d} y \delta_{0}(\mathrm{~d} k),
$$

where, according to (7.16), we have

$$
\begin{aligned}
& e_{\mathrm{th}}^{\prime}(0, y)=e_{\mathrm{th}}^{(0)}(y), \\
& \partial_{t} e_{\mathrm{th}}^{\prime}(t, y)=\hat{c} \partial_{y}^{2} e_{\mathrm{th}}^{\prime}(t, y)+\frac{3 \gamma}{4} \partial_{y}^{2} \overline{\mathfrak{w}}_{\phi}(t, y) \cdot \mathrm{e}+12 \gamma \pi^{2} \int_{\mathbb{R}} h^{2} \mathfrak{W}_{\phi}(t, y, h) \cdot \mathrm{e} \mathrm{d} h .
\end{aligned}
$$

Here $\overline{\mathfrak{w}}_{\phi}(t, y)$ is defined in (7.15),

$$
\mathfrak{W}_{\phi}^{T}(t, y, k):=\left[W_{\phi,+}(t, y, k), Y_{\phi,+}(t, y, k), Y_{\phi,-}(t, y, k), W_{\phi,-}(t, y, k)\right]
$$

and

$$
\begin{aligned}
W_{\phi, \pm}(t, y, k) & =\int_{\mathbb{R}} e^{2 \pi i y \eta} \widehat{W}_{\phi, \pm}(t, \eta, k) \mathrm{d} \eta, \quad Y_{\phi, \pm}(t, y, k) \\
& =\int_{\mathbb{R}} e^{2 \pi i y \eta} \widehat{Y}_{\phi, \pm}(t, \eta, k) \mathrm{d} \eta
\end{aligned}
$$


with $\widehat{W}_{\phi, \pm}$ and $\widehat{Y}_{\phi, \pm}$ given by (7.14). An elementary calculation yields the following.

Proposition 7.4. Suppose that $\phi(t, y)$ is given by (5.25). Then,

$$
\begin{aligned}
& \int_{\mathbb{R}} W_{\phi, \pm}(t, y, h) \mathrm{d} h=\frac{1}{2}|\phi(t, y)|^{2}, \\
& \int_{\mathbb{R}} Y_{\phi,+}(t, y, h) \mathrm{d} h= \frac{1}{2}[\phi(t, y)]^{2}, \\
& 4 \pi^{2} \int_{\mathbb{R}} h^{2} W_{\phi, \pm}(t, y, h) \mathrm{d} h=\frac{1}{4}\left|\phi^{\prime}(t, y)\right|^{2}-\frac{1}{8}\left[\phi(t, y)\left(\phi^{\prime \prime}\right)^{*}(t, y)\right.\left.+\phi^{\prime \prime}(t, y) \phi^{*}(t, y)\right], \\
& 4 \pi^{2} \int_{\mathbb{R}} h^{2} Y_{\phi,+}(t, y, h) \mathrm{d} h=\frac{1}{4}\left\{\left[\phi^{\prime}(t, y)\right]^{2}-\phi(t, y) \phi^{\prime \prime}(t, y)\right\}
\end{aligned}
$$

for any $(t, y) \in[0,+\infty) \times \mathbb{R}$.

Using the proposition we conclude that the third term appearing in the right hand side of the second equation of (7.19) equals

$$
\begin{aligned}
& 12 \gamma \pi^{2} \int_{\mathbb{R}} h^{2}\left(W_{\phi,+}(t, y, h)-\operatorname{Re} Y_{\phi,+}(t, y, h)\right) \mathrm{d} h \\
& =\frac{3 \gamma}{2}\left[\left(p^{\prime}\right)^{2}(t, y)-p(t, y) p^{\prime \prime}(t, y)\right] .
\end{aligned}
$$

On the other hand, the second term equals

$$
\begin{aligned}
& \frac{3 \gamma}{4} \partial_{y}^{2}\left\{\int_{\mathbb{R}}\left(W_{\phi,+}(t, y, h)-\operatorname{Re} Y_{\phi,+}(t, y, h)\right) \mathrm{d} h\right\}=\frac{3 \gamma}{4} \partial_{y}^{2} p^{2}(t, y) \\
& \quad=\frac{3 \gamma}{2}\left\{\left(p^{\prime}\right)^{2}(t, y)+p(t, y) p^{\prime \prime}(t, y)\right\} .
\end{aligned}
$$

We can see therefore that $e_{\mathrm{th}}^{\prime}(t, y)$ satisfies (3.7). Thus the conclusion of Theorem 5.1 follows.

\section{Proof of Theorem 7.1}

We start with the following result.

Lemma 8.1. For any $M>0$ and $\epsilon_{0}, \lambda_{0}$ as in Proposition 6.1 we have

$$
\frac{R}{\tilde{\delta}_{\epsilon}} \sum_{j}\left|\tilde{d}_{j}^{(\epsilon)}\right| \preceq 1, \quad \forall k \in \mathbb{T}, \quad|\eta| \leq M, \quad \epsilon \in\left(0, \epsilon_{0}\right], \quad \lambda>\lambda_{0} .
$$

The summation extends over $j \in\{1,2, o,-,+\}$. 
Proof. From the definition of $\tilde{D}_{1}^{(\epsilon)}$, see (6.13), we obtain

$$
\left|\tilde{D}_{1}^{(\epsilon)}\right| \preceq R_{\epsilon}+\lambda \epsilon^{2}+\epsilon\left|\delta_{\epsilon} \omega\right|
$$

for $k \in \mathbb{T},|\eta| \leq M, \epsilon \in\left(0, \epsilon_{0}\right], \lambda>\lambda_{0}$. Using the first formula of (6.3) we conclude that then

$$
\left|\tilde{D}_{1}^{(\epsilon)}\right| \preceq R_{\epsilon}+\lambda \epsilon^{2} .
$$

A similar consideration leads to the estimate

$$
\left|\tilde{D}_{j}^{(\epsilon)}\right| \preceq R_{\epsilon}+\lambda \epsilon^{2}, \quad k \in \mathbb{T}, \quad|\eta| \leq M, \quad \epsilon \in\left(0, \epsilon_{0}\right], \quad \lambda>\lambda_{0}
$$

for any $j \in\{1,2,+,-\}$. In particular we can conclude from (6.39) that

$$
\sum_{j}\left|\tilde{d}_{j}^{(\epsilon)}\right| \preceq\left(R_{\epsilon}+\lambda \epsilon^{2}\right)^{3} \asymp \sum_{j=0}^{3} R_{\epsilon}^{3-j}\left(\lambda \epsilon^{2}\right)^{j} .
$$

Thanks to (6.28) we infer that

$$
R\left|\tilde{d}_{j}^{(\epsilon)}\right| \preceq\left(R_{\epsilon}+\lambda \epsilon^{2}\right)^{4} \preceq \tilde{\delta}_{\epsilon}, \quad j \in\{1,2, o,+,-\} .
$$

\subsection{Proof of (7.9)}

We show (7.9) for $\left(\iota, \iota^{\prime}\right)=(-,+)$. The cases of other values of $\left(\iota, \iota^{\prime}\right)$ can be handled in the same way. We use the second equation of the system (7.6). Estimate in question follows, provided we can show that the left hand side of the equation can be written in the form

$$
\epsilon^{-2} y_{\epsilon}^{(-)}(\lambda, \eta)+\tilde{T}_{\epsilon}(\lambda, \eta)=z_{\epsilon}^{(2,-)}(\lambda, \eta),
$$

where

$$
\begin{aligned}
& \tilde{T}_{\epsilon}(\lambda, \eta)=O(1), \\
& z_{\epsilon}^{(2,-)}(\lambda, \eta)=O(1), \quad \text { as } \epsilon \ll 1 .
\end{aligned}
$$

We can write

$$
\tilde{T}_{\epsilon}(\lambda, \eta)=-2 b_{o}^{(\epsilon)} v_{\epsilon}^{(-)}-2 b_{-}^{(\epsilon)} v_{\epsilon}^{(+)},
$$

where $v_{\epsilon}^{( \pm)}(\lambda, \eta):=\left\langle v_{\epsilon}(\lambda, \eta, \cdot), \mathfrak{e}_{ \pm}\right\rangle($see $(6.17))$ and

$$
\begin{aligned}
b_{o}^{(\epsilon)} & :=-\frac{3 \gamma}{4 \epsilon^{2}} \int_{\mathbb{T}} \mathfrak{e}_{-} \mathfrak{e}_{+} \frac{\tilde{\Delta}_{2, \epsilon} \mathrm{d} k}{\tilde{\delta}_{\epsilon}}, \\
b_{ \pm}^{(\epsilon)} & :=-\frac{3 \gamma}{4 \epsilon^{2}} \int_{\mathbb{T}} \mathfrak{e}_{ \pm}^{2} \frac{\tilde{\Delta}_{2, \epsilon} \mathrm{d} k}{\tilde{\delta}_{\epsilon}} .
\end{aligned}
$$


Substituting from (6.13) into (7.5) we find

$$
\begin{aligned}
\epsilon^{-2} \tilde{\Delta}_{2, \epsilon}= & \delta_{\epsilon} \omega\left[2 \eta \bar{\omega} R^{\prime}-2\left(\gamma R_{\epsilon}\right) \delta_{\epsilon} \omega\right] \\
& -4 \lambda\left(\gamma R_{\epsilon}\right)\left[\left(\gamma R_{\epsilon}\right)+\epsilon^{2} \lambda\right]+\epsilon^{2} \lambda\left[\left(\delta_{\epsilon} \omega\right)^{2}-\left(R^{\prime} \eta\right)^{2}\right]+\epsilon^{4} \lambda^{3} \\
& +i\left\{4 \lambda \bar{\omega}\left(\gamma R_{\epsilon}\right)+\epsilon^{2} \lambda \delta_{\epsilon} \omega R^{\prime} \eta+2 \epsilon^{2} \bar{\omega}\left(\delta_{\epsilon} \omega\right)^{2}+2 \epsilon^{2} \lambda^{2} \bar{\omega}\right\} .
\end{aligned}
$$

Therefore, $\mathrm{cf}$ (6.27), we conclude that

$$
\epsilon^{-2}\left|\tilde{\Delta}_{2, \epsilon}\right|\left(\mathfrak{e}_{-} \mathfrak{e}_{+}+\mathfrak{e}_{-}^{2}+\mathfrak{e}_{+}^{2}\right) \preceq \epsilon^{-2}\left|\tilde{\Delta}_{2, \epsilon}\right| R^{2} \preceq \tilde{\delta}_{\epsilon}^{(0)}
$$

Thus,

$$
\left|b_{o}^{(\epsilon)}\right|+\left|b_{-}^{(\epsilon)}\right|+\left|b_{+}^{(\epsilon)}\right| \preceq 1
$$

and the first equality of (8.7) follows.

Since (see (6.16))

$$
f_{\epsilon}^{*}(\lambda,-\eta, k)=f_{\epsilon}(\lambda, \eta,-k)=f_{\epsilon}(\lambda, \eta, k)
$$

the right hand side of the second equation of system (7.6) can be written as

$$
z_{\epsilon}^{(2,-)}=Z_{\epsilon, 1}+Z_{\epsilon, 2}+Z_{\epsilon, 3},
$$

where $\left(\mathfrak{d}_{j, \epsilon}\right.$ are the rows of the adjugate matrix to $\tilde{D}_{\epsilon}$, given by (6.38))

$$
\begin{aligned}
& Z_{\epsilon, 1}:=\int_{\mathbb{T}} \mathfrak{d}_{2, \epsilon} \cdot \widehat{\mathfrak{W}}_{\epsilon} \frac{\mathfrak{e}_{-} \mathrm{d} k}{\tilde{\delta}_{\epsilon}}, \\
& Z_{\epsilon, 2}:=-\frac{\gamma(\pi \eta)^{2}}{2} \int_{\mathbb{T}} \tilde{\Delta}_{2, \epsilon} \frac{\mathfrak{e}_{-} f_{\epsilon}}{\tilde{\delta}_{\epsilon}} \mathrm{d} k, \\
& Z_{\epsilon, 3}:=\epsilon \int_{\mathbb{T}} \mathfrak{d}_{2, \epsilon} \cdot \mathfrak{r}_{\epsilon} \frac{\mathfrak{e}_{-} \mathrm{d} k}{\tilde{\delta}_{\epsilon}} .
\end{aligned}
$$

We can write

$$
\left|Z_{\epsilon, 1}\right| \leq\left(\sum_{j} \sup _{k} \frac{\mathfrak{e}_{-}\left|\tilde{d}_{j}^{(\epsilon)}\right|}{\left|\tilde{\delta}_{\epsilon}\right|}\right) \sum_{\iota= \pm}\left(\left\|\widehat{W}_{\epsilon, \iota}\right\|_{\mathcal{A}^{\prime}}+\left\|\widehat{Y}_{\epsilon, l}\right\|_{\mathcal{A}^{\prime}}\right)
$$

Using Lemma 8.1 we conclude that for any $M>0$ there exists $\lambda_{0}$ such that for any $\lambda>\lambda_{0}$ we have $\left|Z_{\epsilon, 1}\right|=O(1)$, as $\epsilon \ll 1$. A similar argument allows us to conclude that also $\left|Z_{\epsilon, j}\right|=O(1)$, as $\epsilon \ll 1$ for $j=2,3$. Thus, the second equality in (8.7) follows as well. 


\subsection{Proof of (7.8)}

The left hand side of the first equation of the system (7.6) can be rewritten in the following form

$$
a_{w,-}^{(\epsilon)} w_{\epsilon}^{(-)}-a_{-}^{(\epsilon)} \frac{\delta w_{\epsilon}}{\epsilon^{2}}+a_{o}^{(\epsilon)} \frac{y_{\epsilon, o}^{(-)}}{\epsilon^{2}}+a_{-}^{(\epsilon)} \frac{y_{\epsilon, o}^{(+)}}{\epsilon^{2}}
$$

with

$$
\begin{aligned}
& a_{w, \pm}^{(\epsilon)}:=\epsilon^{-2}\left[1-2 \int_{\mathbb{T}}(\gamma R) \mathfrak{e}_{ \pm} \frac{\tilde{\Delta}_{1, \epsilon}}{\tilde{\delta}_{\epsilon}} \mathrm{d} k\right], \\
& a_{o}^{(\epsilon)}:=\frac{3 \gamma}{4} \int_{\mathbb{T}} \mathfrak{e}_{-} \mathfrak{e}_{+} \frac{\tilde{\Delta}_{1, \epsilon}}{\tilde{\delta}_{\epsilon}} \mathrm{d} k, \\
& a_{ \pm}^{(\epsilon)}:=\frac{3 \gamma}{4} \int_{\mathbb{T}} \mathfrak{e}_{ \pm}^{2} \frac{\tilde{\Delta}_{1, \epsilon}}{\tilde{\delta}_{\epsilon}} \mathrm{d} k .
\end{aligned}
$$

Note that $\mathfrak{e}_{-} \mathfrak{e}_{+} \preceq R^{3}$ (see (6.6)). From Lemma 8.1 and the Lebesgue dominated convergence theorem we conclude that $a_{o}^{(\epsilon)}$ and $a_{ \pm}^{(\epsilon)}$ are of order $O(1)$, as $\epsilon \ll 1$.

Using (7.5) together with formulas (6.40) we infer that

$$
\tilde{\Delta}_{1, \epsilon}=8 \gamma R_{\epsilon} \bar{\omega}^{2}+o(1), \quad \text { as } \epsilon \ll 1 .
$$

Therefore, by the Lebesgue dominated convergence theorem

$$
\lim _{\epsilon \rightarrow 0+} a_{o}^{(\epsilon)}=\bar{a}_{o}:=\frac{3}{8} \int_{\mathbb{T}} \frac{\mathfrak{e}_{-} \mathfrak{e}_{+} \mathrm{d} k}{R}>0
$$

and

$$
\lim _{\epsilon \rightarrow 0+} a_{ \pm}^{(\epsilon)}=\bar{a}_{ \pm}:=\frac{3}{8} \int_{\mathbb{T}} \frac{\mathfrak{e}_{ \pm}^{2} \mathrm{~d} k}{R}>0
$$

After a direct computation we obtain

$$
a_{w,-}^{(\epsilon)}=\int_{\mathbb{T}} \mathfrak{e}_{-} \frac{\tilde{e}_{\epsilon}}{\tilde{\delta}_{\epsilon}} \mathrm{d} k
$$

where

$$
\begin{aligned}
\tilde{e}_{\epsilon}:= & \left\{4(\gamma R) \eta \delta_{\epsilon} \omega \bar{\omega} R^{\prime}+2 \eta^{2} \gamma R^{\prime \prime}\left(\gamma R_{\epsilon}\right) \bar{\omega}^{2}+4\left[\left(\gamma R_{\epsilon} \delta_{\epsilon} \omega\right)^{2}\right.\right. \\
& \left.\left.-2\left(\gamma R_{\epsilon}\right) \delta_{\epsilon} \omega \bar{\omega} \hat{R}^{\prime} \eta+\left(\bar{\omega} \delta_{\epsilon} \omega\right)^{2}\right]\right\} \\
& +4 \lambda\left(\gamma R_{\epsilon}\right)\left[4\left(\gamma R_{\epsilon}\right)^{2}+4 \bar{\omega}^{2}+\left(\epsilon \delta_{\epsilon} \omega\right)^{2}-\left(\epsilon \hat{R}^{\prime} \eta\right)^{2}\right] \\
& -4(\gamma R) \lambda\left[2\left(\gamma R_{\epsilon}\right)^{2}+2 \bar{\omega}^{2}-2\left(\frac{\epsilon R^{\prime} \eta}{2}\right)^{2}\right] \\
& +4 \epsilon^{2}\left\{\lambda^{2}\left[5\left(\gamma R_{\epsilon}\right)^{2}+\bar{\omega}^{2}+\left(\epsilon \delta_{\epsilon} \omega\right)^{2}-\left(\frac{\epsilon \hat{R}^{\prime} \eta}{2}\right)^{2}\right]-2 \gamma R \lambda^{2}\left(\gamma R_{\epsilon}\right)\right\} \\
& -2(\gamma R) \lambda^{3} \epsilon^{4}+8 \epsilon^{4} \lambda^{3} \gamma R_{\epsilon}+\epsilon^{6} \lambda^{4} .
\end{aligned}
$$


Taking into account (6.28) we conclude that $\mathfrak{e}_{-} \tilde{e}_{\epsilon} \preceq \tilde{\delta}_{\epsilon}$. In addition,

$$
\tilde{e}_{\epsilon}=2 \eta^{2} \gamma R^{\prime \prime}\left(\gamma R_{\epsilon}\right) \bar{\omega}^{2}+8 \lambda\left(\gamma R_{\epsilon}\right)\left[\left(\gamma R_{\epsilon}\right)^{2}+\bar{\omega}^{2}\right]+o(1), \quad \text { as } \epsilon \ll 1 .
$$

Combining this with the second formula of (8.18) we obtain, by the Lebesgue dominated convergence theorem,

$$
\bar{a}:=\lim _{\epsilon \rightarrow 0+} \gamma a_{w,-}^{(\epsilon)}<+\infty .
$$

Using the above together with bound (7.9) we conclude that expression (8.16) can be written as

$$
\frac{\bar{a}}{\gamma}[1+o(1)] w_{\epsilon}^{(-)}-\bar{a}_{-}[1+o(1)] \frac{\delta w_{\epsilon}}{\epsilon^{2}}+O(1), \quad \text { as } \epsilon \ll 1 .
$$

Then bound (7.8) would follow, provided we can show that the right hand side of the first equation of the system (7.6), given by $z_{\epsilon}^{(1,-)}$, is of order of magnitude $O(1)$, as $\epsilon \ll 1$. To see that we write

$$
z_{\epsilon}^{(1,-)}=U_{\epsilon, 1}+U_{\epsilon, 2}+U_{\epsilon, 3},
$$

where the terms $U_{\epsilon, i}, i=1,2,3$ are given by

$$
\begin{aligned}
U_{\epsilon, 1} & =\int_{\mathbb{T}} \mathfrak{d}_{1, \epsilon} \cdot \widehat{\mathfrak{W}}_{\epsilon} \frac{\mathfrak{e}_{-} \mathrm{d} k}{\tilde{\delta}_{\epsilon}}, \\
U_{\epsilon, 2} & :=-\frac{\gamma(\pi \eta)^{2}}{2} \int_{\mathbb{T}} \tilde{\Delta}_{1, \epsilon} \frac{\mathfrak{e}_{-} f_{\epsilon}}{\tilde{\delta}_{\epsilon}} \mathrm{d} k, \\
U_{\epsilon, 3} & :=\epsilon \int_{\mathbb{T}} \mathfrak{d}_{1, \epsilon} \cdot \mathfrak{r}_{\epsilon} \frac{\mathfrak{e}_{-} \mathrm{d} k}{\tilde{\delta}_{\epsilon}} .
\end{aligned}
$$

The fact that $z_{\epsilon}^{(1,-)}=O(1)$, as $\epsilon \ll 1$, can be argued in a similar way as it has been done in the case of $z_{\epsilon}^{(2,-)}$, see (8.14) and (8.15) above.

\subsection{Proof of (7.10)}

From (6.20) we obtain

$$
w_{\epsilon,+}(\lambda, \eta, k)=I_{\epsilon}+I I_{\epsilon}+I I I_{\epsilon}+I V_{\epsilon},
$$

where

$$
\begin{aligned}
I_{\epsilon}:= & \frac{3 \gamma}{2 \tilde{\delta}_{\epsilon}} \tilde{\Delta}_{1}^{(\epsilon)} \sum_{\iota \in\{-,+\}}\left\langle v_{\epsilon}, \mathfrak{e}_{\iota}\right\rangle_{L^{2}(\mathbb{T})^{\mathfrak{e}_{-\iota}}}, \\
I I_{\epsilon}:= & -\frac{\gamma(\pi \epsilon \eta)^{2}}{2 \tilde{\delta}_{\epsilon}} \tilde{\Delta}_{1}^{(\epsilon)}\left[\mathfrak{f}_{+}\left\langle v_{\epsilon}, 16 \mathfrak{f}_{+}+\mathfrak{e}_{-}\right\rangle_{L^{2}(\mathbb{T})}+\mathfrak{e}_{-}\left\langle v_{\epsilon}, \mathfrak{f}_{+}\right\rangle_{L^{2}(\mathbb{T})}\right. \\
& \left.+3 \tilde{f}_{-}\left\langle v_{\epsilon}, \mathfrak{e}_{+}\right\rangle_{L^{2}(\mathbb{T})}+3 \mathfrak{e}_{+}\left\langle v_{\epsilon}, \mathfrak{f}_{-}\right\rangle_{L^{2}(\mathbb{T})}\right] \\
I I I_{\epsilon}:= & \epsilon^{2} \tilde{\delta}_{\epsilon}^{-1} \mathfrak{d}_{\epsilon, 1} \cdot \widehat{\mathfrak{W}}_{\epsilon}, \quad I V_{\epsilon}:=\epsilon^{3} \tilde{\delta}_{\epsilon}^{-1} \mathfrak{d}_{\epsilon, 1} \cdot \mathfrak{r}_{\epsilon} .
\end{aligned}
$$


8.3.1. Convergence of $I_{\epsilon}$ Note that, (see (6.26)) for any $k \neq 0$

$$
\lim _{\epsilon \rightarrow+0} \frac{(\gamma R) \tilde{\Delta}_{1}^{(\epsilon)}}{\tilde{\delta}_{\epsilon}}=\frac{1}{2} .
$$

Using the above and the already proved estimates (7.8), (7.9) and Lemma 8.1 we obtain

$$
\lim _{\epsilon \rightarrow 0+}\left\|I_{\epsilon}-w_{\epsilon}^{(-)} \mathfrak{f}\right\|_{L^{1}(\mathbb{T})}=0
$$

Here $\mathfrak{f}(k) \equiv 1$.

8.3.2. Convergence of $I I_{\epsilon}, I I I_{\epsilon}$ and $I V_{\epsilon}$ Thanks to Lemma 8.1 we can write

$$
\epsilon^{2} \int_{\mathbb{T}}\left|\tilde{\delta}_{\epsilon}^{-1} \widehat{W}_{\epsilon} \tilde{d}_{1}^{(\epsilon)}\right| R \mathrm{~d} k \preceq \epsilon^{2}\left\|\widehat{W}_{\epsilon}\right\|_{\mathcal{A}^{\prime}} \rightarrow 0, \quad \text { as } \quad \epsilon \rightarrow 0+
$$

The remaining terms appearing in expressions $I I_{\epsilon}, I I I_{\epsilon}$ and $I V_{\epsilon}$ can be estimated in the same manner allowing us to conclude that

$$
\lim _{\epsilon \rightarrow 0+} \int_{\mathbb{T}}\left(\left|I I_{\epsilon}\right|+\left|I I I_{\epsilon}\right|+\left|I V_{\epsilon}\right|\right) R \mathrm{~d} k=0 .
$$

\subsection{Proof of (7.11)}

From (6.20) we obtain

$$
y_{\epsilon}(\lambda, \eta, k)=I_{\epsilon}+I I_{\epsilon}+I I I_{\epsilon}+I V_{\epsilon},
$$

where

$$
\begin{aligned}
I_{\epsilon}:= & \frac{3 \gamma}{2 \tilde{\delta}_{\epsilon}} \tilde{\Delta}_{2}^{(\epsilon)} \sum_{\iota \in\{-,+\}}\left\langle v_{\epsilon}, \mathfrak{e}_{\iota}\right\rangle_{L^{2}(\mathbb{T})} \mathfrak{e}_{-\iota}, \\
I I_{\epsilon}:= & -\frac{\gamma(\pi \epsilon \eta)^{2}}{2 \tilde{\delta}_{\epsilon}} \tilde{\Delta}_{2}^{(\epsilon)}\left[\mathfrak{f}_{+}\left\langle v_{\epsilon}, 16 \mathfrak{f}_{+}+\mathfrak{e}_{-}\right\rangle_{L^{2}(\mathbb{T})}+\mathfrak{e}_{-}\left\langle v_{\epsilon}, \mathfrak{f}_{+}\right\rangle_{L^{2}(\mathbb{T})}\right. \\
& \left.+3 \mathfrak{f}_{-}\left\langle v_{\epsilon}, \mathfrak{e}_{+}\right\rangle_{L^{2}(\mathbb{T})}+3 \mathfrak{e}_{+}\left\langle v_{\epsilon}, \mathfrak{f}_{-}\right\rangle_{L^{2}(\mathbb{T})}\right] \\
I I I_{\epsilon}:= & \epsilon^{2} \tilde{\delta}_{\epsilon}^{-1} \mathfrak{d}_{\epsilon, 2} \cdot \widehat{\mathfrak{W}}_{\epsilon}, \quad I V_{\epsilon}:=\epsilon^{3} \tilde{\delta}_{\epsilon}^{-1} \mathfrak{d}_{\epsilon, 2} \cdot \mathfrak{r}_{\epsilon} .
\end{aligned}
$$

The analysis of the above terms is very similar to what has been done in the precious section. Using (8.10) we conclude that for any $\lambda>\lambda_{0}$

$$
\left|\frac{\tilde{\Delta}_{2}^{(\epsilon)}(\lambda, \eta, k)}{\tilde{\delta}_{\epsilon}(\lambda, \eta, k)}\right| \preceq \epsilon^{2}, \quad k \in \mathbb{T},|\eta| \leq M .
$$

We conclude in this way that all $R I_{\epsilon}, R I I_{\epsilon}, R I I I_{\epsilon}$ and $R I V_{\epsilon}$ tend to 0 in the $L^{1}$ sense. Thus, (7.11) follows. 


\section{Proof of Theorem 7.2}

\subsection{Determining $w_{\epsilon}^{(-)}$}

Since functions $\mathfrak{e}_{ \pm}(k)$ are both even the fourth and eighth equation of the system (7.6) coincide with the first and the fifth ones respectively.

Adding the first and fifth equations of the system (7.6) we get

$$
\gamma a_{w}^{(\epsilon)} w_{\epsilon}^{(-)}-\sum_{\iota \in\{-,+\}} a_{y,-\iota}^{(\epsilon)} \hat{y}_{\epsilon, o}^{(\iota)}=\gamma z_{\epsilon}^{(1, o)}+\gamma a_{w,-}^{(\epsilon)} \delta w_{\epsilon} .
$$

Here $a_{w, \iota}^{(\epsilon)}, a_{\iota}^{(\epsilon)}, \iota \in\{o,-,+\}$ are given by (8.17) and $\hat{y}_{\epsilon, o}^{( \pm)}:=\epsilon^{-2} y_{\epsilon, o}^{( \pm)}$. In addition

$$
\begin{aligned}
& a_{w}^{(\epsilon)}:=a_{w,-}^{(\epsilon)}+a_{w,+}^{(\epsilon)}=\frac{4}{3 \epsilon^{2}} \int_{\mathbb{T}} \gamma R\left[1-2(\gamma R) \frac{\tilde{\Delta}_{1, \epsilon}}{\tilde{\delta}_{\epsilon}}\right] \mathrm{d} k, \\
& a_{y, \pm}^{(\epsilon)}:=a_{ \pm}^{(\epsilon)}+a_{o}^{(\epsilon)}:=\int_{\mathbb{T}} \mathfrak{e}_{ \pm} \tilde{\Delta}_{1, \epsilon} \frac{\gamma R \mathrm{~d} k}{\tilde{\delta}_{\epsilon}}
\end{aligned}
$$

and

$$
z_{\epsilon}^{(1, o)}:=z_{\epsilon}^{(1,-)}+z_{\epsilon}^{(1,+)}
$$

where $z_{\epsilon}^{(1, \pm)}$ are the scalar products of $z_{\epsilon}^{(1)}$ by $\mathfrak{e}_{ \pm}(\mathrm{cf}(7.3)$ and (7.7)).

The second and third equations of (7.6) read (cf (8.9))

$$
\begin{aligned}
& \gamma b_{o}^{(\epsilon)} w_{\epsilon}^{(-)}+\gamma b_{-}^{(\epsilon)} w_{\epsilon}^{(+)}+\hat{y}_{\epsilon,+}^{(-)}=\gamma z_{\epsilon,+}^{(2,-)}-\epsilon^{2}\left[b_{o}^{(\epsilon)} \hat{y}_{\epsilon, o}^{(-)}+b_{-}^{(\epsilon)} \hat{y}_{\epsilon, o}^{(+)}\right], \\
& \gamma\left(b_{o}^{(\epsilon)}\right)^{*} w_{\epsilon}^{(-)}+\gamma\left(b_{-}^{(\epsilon)}\right)^{*} w_{\epsilon}^{(+)}+\hat{y}_{\epsilon,+}^{(-)}=\gamma z_{\epsilon,-}^{(2,-)}-\epsilon^{2}\left[\left(b_{o}^{(\epsilon)}\right)^{*} \hat{y}_{\epsilon, o}^{(-)}+\left(b_{-}^{(\epsilon)}\right)^{*} \hat{y}_{\epsilon, o}^{(+)}\right] .
\end{aligned}
$$

Adding these equations sideways we get

$$
2 \gamma w_{\epsilon}^{(-)} \operatorname{Re} b_{o}^{(\epsilon)}+2 \gamma w_{\epsilon}^{(+)} \operatorname{Re} b_{-}^{(\epsilon)}+\hat{y}_{\epsilon, o}^{(-)}=\gamma z_{\epsilon, o}^{(2,-)}+r_{\epsilon}^{(-)} .
$$

Here $z_{\epsilon, o}^{(2, \pm)}:=z_{\epsilon}^{(2, \pm)}+z_{\epsilon,-}^{(2, \pm)}$ and

$$
r_{\epsilon}^{(-)}:=-2 \epsilon^{2}\left(\hat{y}_{\epsilon, o}^{(-)} \operatorname{Re} b_{o}^{(\epsilon)}+\hat{y}_{\epsilon, o}^{(+)} \operatorname{Re} b_{-}^{(\epsilon)}\right)
$$

The sixth and seventh equations of (7.6) yield

$$
2 \gamma w_{\epsilon}^{(-)} \operatorname{Re} b_{+}^{(\epsilon)}+2 \gamma w_{\epsilon}^{(+)} \operatorname{Re} b_{o}^{(\epsilon)}+\hat{y}_{\epsilon, o}^{(+)}=\gamma z_{\epsilon, o}^{(2,+)}+r_{\epsilon}^{(+)},
$$

and

$$
r_{\epsilon}^{(+)}:=-2 \epsilon^{2}\left(\hat{y}_{\epsilon, o}^{(-)} \operatorname{Re} b_{+}^{(\epsilon)}+\hat{y}_{\epsilon, o}^{(+)} \operatorname{Re} b_{o}^{(\epsilon)}\right) .
$$


Summarizing, we have obtained the following system

$$
\begin{aligned}
& \gamma a_{w}^{(\epsilon)} w_{\epsilon}^{(-)}-\sum_{\iota \in\{-,+\}} a_{y,-\iota}^{(\epsilon)} \hat{y}_{\epsilon, o}^{(\iota)}=\gamma z_{\epsilon}^{(1, o)}+\gamma a_{w,-}^{(\epsilon)} \delta w_{\epsilon}, \\
& 2 \gamma w_{\epsilon}^{(-)} \operatorname{Re} b_{o}^{(\epsilon)}+2 \gamma w_{\epsilon}^{(+)} \operatorname{Re} b_{-}^{(\epsilon)}+\hat{y}_{\epsilon, o}^{(-)}=\gamma z_{\epsilon, o}^{(2,-)}+r_{\epsilon}^{(-)}, \\
& 2 \gamma w_{\epsilon}^{(-)} \operatorname{Re} b_{+}^{(\epsilon)}+2 \gamma w_{\epsilon}^{(+)} \operatorname{Re} b_{o}^{(\epsilon)}+\hat{y}_{\epsilon, o}^{(+)}=\gamma z_{\epsilon, o}^{(2,+)}+r_{\epsilon}^{(+)} .
\end{aligned}
$$

Using Theorem 7.1 we conclude that given $M>0$ and $\lambda>\lambda_{0}$ the family $\left(w_{\epsilon}^{(-)}, \hat{y}_{\epsilon, o}^{(-)}, \hat{y}_{\epsilon, o}^{(+)}\right)$remains bounded in $L^{\infty}[-M, M]$, as $\epsilon \rightarrow 0+$. It is therefore *-weakly sequentially compact in this space. Denote by

$$
\left(w^{(-)}, \hat{y}_{o}^{(-)}, \hat{y}_{o}^{(+)}\right)
$$

its $*$-weak limit. Thanks to (8.19), (8.12) and the results of Theorem 7.1 we conclude that

$$
\begin{aligned}
& \lim _{\epsilon \rightarrow 0+} \gamma a_{w,-}^{(\epsilon)} \delta w_{\epsilon}=0, \\
& \lim _{\epsilon \rightarrow 0+} \gamma\left(\sum_{l=o, \pm} b_{\iota}^{(\epsilon)}\right) \delta w_{\epsilon}=0, \\
& \lim _{\epsilon \rightarrow 0+} r_{\epsilon}^{( \pm)}=0 .
\end{aligned}
$$

Using Lemma 8.1, equalities (6.40) and the Lebesgue dominated convergence theorem we conclude that

$$
\lim _{\epsilon \rightarrow 0+} a_{y, \iota}^{(\epsilon)}=-\frac{1}{2}, \quad \iota \in\{-,+\}
$$

Subtracting sideways from the first equation of (9.8) the sum of the remaining two and taking into account (9.10) and (9.11), we obtain

$$
\lim _{\epsilon \rightarrow 0+} \gamma\left\{\left(a_{w}^{(\epsilon)}-2 \gamma \sum_{\iota= \pm} \operatorname{Re} b_{w, l}^{(\epsilon)}\right) w_{\epsilon}^{(-)}-\left(z_{\epsilon}^{(1, o)}-\frac{z_{\epsilon, o}^{(2, o)}}{2}\right)\right\}=0
$$

where $z_{\epsilon, o}^{(2, o)}:=z_{\epsilon, o}^{(2,-)}+z_{\epsilon, o}^{(2,+)}$. Moreover, a direct calculation shows that

$$
\gamma\left(a_{w}^{(\epsilon)}-2 \gamma \sum_{\iota= \pm} \operatorname{Re} b_{w, \iota}^{(\epsilon)}\right)=\frac{4 \gamma}{3} \int_{\mathbb{T}} \frac{R \tilde{f}_{\epsilon}}{\tilde{\delta}_{\epsilon}} \mathrm{d} k
$$


where

$$
\begin{aligned}
\tilde{f}_{\epsilon}:= & 4 \gamma\left(R-R_{\epsilon}\right) \eta \delta_{\epsilon} \omega \bar{\omega} R^{\prime}+2 \gamma \eta^{2} R^{\prime \prime}(k)\left(\gamma R_{\epsilon}\right) \bar{\omega}^{2} \\
& +4\left[\gamma\left(R-R_{\epsilon}\right)\left(\gamma R_{\epsilon}\right)\left(\delta_{\epsilon} \omega\right)^{2}+\left(\bar{\omega} \delta_{\epsilon} \omega\right)^{2}\right]-4(\gamma R) \lambda\left[2 \bar{\omega}^{2}-2\left(\frac{\epsilon R^{\prime} \eta}{2}\right)^{2}\right] \\
& +4 \lambda\left(\gamma R_{\epsilon}\right)\left[4 \gamma\left(R-R_{\epsilon}\right)\left(\gamma R_{\epsilon}\right)+4 \bar{\omega}^{2}+\left(\epsilon \delta_{\epsilon} \omega\right)^{2}-\left(\epsilon R^{\prime} \eta\right)^{2}\right] \\
& +4 \epsilon^{2}\left\{\frac{\lambda \gamma}{2} R\left(\delta_{\epsilon} \omega\right)^{2}+\lambda^{2}\left[5 \gamma\left(R-R_{\epsilon}\right)\left(\gamma R_{\epsilon}\right)+\bar{\omega}^{2}+\left(\epsilon \delta_{\epsilon} \omega\right)^{2}\right]\right\} \\
& +8 \epsilon^{4} \lambda^{3} \gamma R_{\epsilon}+\epsilon^{6} \lambda^{4} .
\end{aligned}
$$

Using Lemma 8.1 and the Lebesgue dominated convergence theorem we obtain

$$
\lim _{\epsilon \rightarrow 0+} \frac{4 \gamma}{3} \int_{\mathbb{T}} \frac{R \tilde{f}_{\epsilon}}{\tilde{\delta}_{\epsilon}} \mathrm{d} k=\frac{2 \lambda}{3}+\frac{\eta^{2}}{3 \gamma} \int_{\mathbb{T}} \frac{\left(\omega^{\prime}\right)^{2}}{R} \mathrm{~d} k .
$$

Using the above formula and substituting

$$
z_{\epsilon}^{(1, o)}-\frac{z_{\epsilon, o}^{(2, o)}}{2}=\left(\mathfrak{b}_{\epsilon}^{(-)}+\mathfrak{z}_{\epsilon}^{(+)}\right) \cdot \mathrm{e}
$$

(cf (7.3) and (7.7)) we can rewrite (9.12) in the form

$$
\left(\frac{2 \lambda}{3}+\frac{\eta^{2}}{3 \gamma} \int_{\mathbb{T}} \frac{\left(\omega^{\prime}\right)^{2}}{R} \mathrm{~d} k\right) w^{(-)}=\lim _{\epsilon \rightarrow 0+} \sum_{j=1}^{3} V_{\epsilon, j},
$$

where

$$
\begin{aligned}
V_{\epsilon, 1}= & \frac{2 \gamma}{3} \int_{\mathbb{T}} \mathfrak{w}_{\epsilon}^{(0)}(\lambda, \eta, k) \cdot \mathrm{e} R \mathrm{~d} k \\
V_{\epsilon, 2}= & -\frac{2 \gamma(\pi \eta)^{2}}{3} \int_{\mathbb{T}}\left(\tilde{\Delta}_{1, \epsilon}-\tilde{\Delta}_{2, \epsilon}\right) \frac{R f_{\epsilon}}{\tilde{\delta}_{\epsilon}} \mathrm{d} k \\
V_{\epsilon, 3}:= & \gamma \epsilon \int_{\mathbb{T}} \tilde{\Delta}_{1, \epsilon} \frac{R r_{\epsilon}^{(1)}}{\tilde{\delta}_{\epsilon}} \mathrm{d} k+\frac{\gamma \epsilon}{2} \int_{\mathbb{T}}\left(2 \tilde{\mathrm{d}}_{-}^{(\epsilon)}-\tilde{\mathrm{d}}_{2}^{(\epsilon)}-\tilde{\mathrm{d}}_{o}^{(\epsilon)}\right) \frac{R r_{\epsilon}^{(2)}}{\tilde{\delta}_{\epsilon}} \mathrm{d} k \\
& +\frac{\gamma \epsilon}{2} \int_{\mathbb{T}}\left[2\left(\tilde{\mathrm{d}}_{+}^{(\epsilon)}\right)^{*}-\tilde{\mathrm{d}}_{o}^{(\epsilon)}-\left(\tilde{\mathrm{d}}_{2}^{(\epsilon)}\right)^{*}\right] \frac{R r_{\epsilon,-}^{(2)}}{\tilde{\delta}_{\epsilon}} \mathrm{d} k .
\end{aligned}
$$

The vector

$$
\left(\mathfrak{w}_{\epsilon}^{(0)}\right)^{T}(\lambda, \eta, k):=\left[w_{\epsilon,+}^{(0)}, y_{\epsilon,+}^{(0)}, y_{\epsilon,-}^{(0)}, w_{\epsilon,-}^{(0)}\right]
$$

appearing in the first formula for $V_{\epsilon, 1}$, is the solution of the system

$$
\tilde{D}_{\epsilon} \mathfrak{w}_{\epsilon}^{(0)}(\lambda, \eta, k)=\widehat{\mathfrak{W}}_{\epsilon}(\eta, k),
$$


where $\widehat{\mathfrak{W}}_{\epsilon}(\eta, k)$ is the column vector of Fourier-Wigner functions corresponding to the initial data, see (5.5). In addition, $f_{\epsilon}$ is given by (6.16) respectively, and $r_{\epsilon}^{(i)}$, $i=1,2$ satisfy (6.18).

Note that the quantity

$$
\frac{1}{\gamma} \int_{\mathbb{T}} \frac{\omega^{\prime}(k)^{2}}{R(k)} \mathrm{d} k=\frac{8 \alpha \pi^{2}}{\gamma} \int_{\mathbb{T}} \frac{\mathfrak{c}^{2}(k)}{1+2 \mathfrak{c}^{2}(k)} \mathrm{d} k=-\frac{2 \pi i \alpha}{\gamma} \int_{C} \frac{(z+1)^{2} \mathrm{~d} z}{z\left(z^{2}+4 z+1\right)},
$$

where $\int_{C}$ is the contour integration on the complex plane over the unit circle $C:=$ $[|z|=1]$ oriented counter-clockwise. The last integral can be computed easily by the method of residues and we conclude that

$$
\frac{1}{\gamma} \int_{\mathbb{T}} \frac{\omega^{\prime}(k)^{2}}{R(k)} \mathrm{d} k=\frac{4 \pi^{2}(\sqrt{3}-1) \alpha}{\gamma \sqrt{3}} .
$$

In what follows, we shall also prove that

$$
\begin{aligned}
\lim _{\epsilon \rightarrow 0+} \sum_{j=1}^{3} \int_{\mathbb{R}} V_{\epsilon, j} \hat{J}^{*}(\eta) \mathrm{d} \eta= & \int_{\mathbb{R}}\left\{8 \gamma \pi^{2} \int_{\mathbb{R}} h^{2} \mathfrak{w}_{\phi}(\lambda, \eta, h) \cdot \mathrm{e} \mathrm{d} h+\frac{2}{3} \hat{e}_{\mathrm{th}}^{(0)}(\eta)\right. \\
& \left.-8 \gamma(\pi \eta)^{2} w^{(-)}-2 \gamma(\pi \eta)^{2} \bar{v}_{\phi}\right\} \hat{J}^{*}(\eta) \mathrm{d} \eta
\end{aligned}
$$

for any $J \in \mathcal{S}(\mathbb{R})$ such that $\hat{J}$ is compactly supported. Here (cf (7.15))

$$
\bar{v}_{\phi}(\lambda, \eta):=\bar{w}_{\phi}(\lambda, \eta)-\frac{1}{2}\left(\bar{y}_{\phi,-}(\lambda, \eta)+\bar{y}_{\phi,+}(\lambda, \eta)\right) .
$$

Combining the above with (9.16) we obtain that $w^{(-)}$satisfies the equation

$$
\left(\lambda+4 \pi^{2} \hat{c} \eta^{2}\right) w^{(-)}=\hat{e}_{\mathrm{th}}^{(0)}(\eta)+12 \gamma \pi^{2} \int_{\mathbb{R}} h^{2} \mathfrak{w}_{\phi}(\lambda, \eta, h) \cdot \mathrm{ed} h-3 \gamma(\pi \eta)^{2} \bar{v}_{\phi},
$$

where $\hat{c}$ is given by (3.8).

9.2. Limits of $V_{\epsilon, j}, j=2,3$

Thanks to Lemma 8.1 we conclude, upon an application of the Lebesgue dominated convergence theorem, that

$$
\lim _{\epsilon \rightarrow 0+} V_{\epsilon, 3}=0
$$

Using Theorems 7.1 and 7.3 and the definition of $f_{\epsilon}$ (see (6.16)) we get

$$
\lim _{\epsilon \rightarrow 0+} V_{\epsilon, 2}=-8 \gamma(\pi \eta)^{2} w^{(-)}-2 \gamma(\pi \eta)^{2} \bar{v}_{\phi}
$$

with $\bar{v}_{\phi}(\lambda, \eta)$ given by (9.20). 


\subsection{Limit of $V_{\epsilon, 1}$}

Using the decomposition of the Wigner functions of the initial data into the parts corresponding to the macroscopic profile and the fluctuations, see (5.13), we can write an analogous decomposition $\widehat{\mathfrak{W}}_{\epsilon}=\widehat{\mathfrak{W}}_{\epsilon}+\widehat{\widetilde{\mathfrak{W}}}_{\epsilon}$, for the LaplaceFourier transforms of the respective Wigner functions. It allows us to write $V_{\epsilon, 1}=$ $V_{\epsilon, 1}^{(1)}+V_{\epsilon, 1}^{(2)}$, where

$$
\begin{aligned}
V_{\epsilon, 1}^{(1)} & :=\frac{2 \gamma}{3} \int_{\mathbb{T}} \overline{\mathfrak{w}}_{\epsilon}^{(0)}(\lambda, \eta, k) \cdot \mathrm{e} R \mathrm{~d} k, \\
V_{\epsilon, 1}^{(2)} & :=\frac{2 \gamma}{3} \int_{\mathbb{T}} \widetilde{\mathfrak{w}}_{\epsilon}^{(0)}(\lambda, \eta, k) \cdot \mathrm{e} R \mathrm{~d} k .
\end{aligned}
$$

Here

$$
\overline{\mathfrak{w}}_{\epsilon}^{(0)}=\left[\bar{w}_{\epsilon,+}^{(0)}, \bar{y}_{\epsilon,+}^{(0)}, \bar{y}_{\epsilon,-}^{(0)}, \bar{w}_{\epsilon,-}^{(0)}\right], \quad \widetilde{\mathfrak{w}}_{\epsilon}^{(0)}=\left[\widetilde{w}_{\epsilon,+}^{(0)}, \widetilde{y}_{\epsilon,+}^{(0)}, \widetilde{y}_{\epsilon,-}^{(0)}, \widetilde{w}_{\epsilon,-}^{(0)}\right]
$$

are the solutions of the analogues of (9.18) in which the right hand side has been replaced by $\widehat{\overline{\mathfrak{W}}}_{\epsilon}$ and $\widehat{\widetilde{\mathfrak{W}}}_{\epsilon}$, respectively.

\subsubsection{Macroscopic Wigner Functions and Their Dynamics. From (3.1) we get}

$$
\partial_{t} \hat{\phi}(t, k)=-i \tau_{2}(\pi k)^{2} \hat{\phi}(t, k)-6 \gamma \pi^{2} k^{2}\left(\hat{\phi}(t, k)-\hat{\phi}^{*}(t,-k)\right) .
$$

Therefore the Fourier transforms $\widehat{\mathfrak{W}}_{\phi}(t)$ of the macroscopic Wigner functions (cf (7.14)) satisfy

$$
\begin{aligned}
\partial_{t} \widehat{W}_{\phi,+}= & -2 \pi^{2}\left\{i \tau_{2} k \eta+6 \gamma\left[k^{2}+\left(\frac{\eta}{2}\right)^{2}\right]\right\} \widehat{W}_{\phi,+} \\
& +6 \gamma \pi^{2} \sum_{\iota^{\prime} \in\{-,+\}}\left(k-\iota^{\prime} \frac{\eta}{2}\right)^{2} \widehat{Y}_{\phi, \iota^{\prime}}, \\
\partial_{t} \widehat{Y}_{\phi,+}= & -2 \pi^{2}\left(i \iota \tau_{2}+6 \gamma\right)\left[k^{2}+\left(\frac{\eta}{2}\right)^{2}\right] \widehat{Y}_{\phi,+}+6 \gamma \pi^{2} \sum_{\iota^{\prime}= \pm 1}\left(k-\iota^{\prime} \frac{\eta}{2}\right)^{2} \widehat{W}_{\phi, \iota^{\prime}} .
\end{aligned}
$$

Taking the Laplace transforms of both sides of (9.25) we obtain

$$
\tilde{D}_{1} w_{\phi,+}+\tilde{D}_{+} y_{\phi,+}+\tilde{D}_{-y_{\phi,-}}=\widehat{W}_{\phi,+}(0, \eta, k)
$$

and

$$
\tilde{D}_{+} w_{\phi,+}+\tilde{D}_{2 y_{\phi,+}}+\tilde{D}_{-} w_{\phi,-}=\widehat{Y}_{\phi,+}(0, \eta, k),
$$

where

$$
\begin{aligned}
& \tilde{D}_{1}:=\lambda+2 \pi^{2}\left\{6 \gamma\left[k^{2}+\left(\frac{\eta}{2}\right)^{2}\right]+i \tau_{2} k \eta\right\}, \\
& \tilde{D}_{2}:=\lambda+2 \pi^{2}\left[k^{2}+\left(\frac{\eta}{2}\right)^{2}\right]\left(6 \gamma+i \tau_{2}\right), \\
& \tilde{D}_{ \pm}:=-6 \gamma \pi^{2}\left(k \mp \frac{\eta}{2}\right)^{2} .
\end{aligned}
$$


An elementary calculation shows that

$$
\lim _{\epsilon \rightarrow 0+} \epsilon^{-2} \tilde{D}_{j}^{(\epsilon)}(\lambda, q, \epsilon k)=\tilde{D}_{j}(\lambda, \eta, k), \quad j \in\{1,2,-,+\}
$$

for any $\lambda>0$ and $(\eta, k) \in \mathbb{R}^{2}$.

The closed system of linear algebraic equations for the components of the Laplace-Fourier transforms $\mathfrak{w}_{\phi}(\mathrm{cf}(7.12))$ takes the form

$$
\tilde{D} \mathfrak{w}_{\phi}=\widehat{\mathfrak{W}}_{\phi}(0)
$$

where

$$
\tilde{D}=\left[\begin{array}{ll}
A & B \\
B & C
\end{array}\right]
$$

with

$$
A=\left[\begin{array}{cc}
\tilde{D}_{1} & \tilde{D}_{+} \\
\tilde{D}_{+} & \tilde{D}_{2}
\end{array}\right], \quad C=\left[\begin{array}{cc}
\tilde{D}_{2}^{*} & \tilde{D}_{+} \\
\tilde{D}_{+} & \tilde{D}_{1}^{*}
\end{array}\right]
$$

and $B=\tilde{D}_{-} I_{2}(\mathrm{cf}(6.21)$ and (6.22)). It can be checked by a direct inspection that $[A, B]=[B, C]=[A, C]=0$. Therefore,

$$
\begin{aligned}
\tilde{\delta}(\lambda, \eta, k) & :=\operatorname{det} \tilde{D}(\lambda, \eta, k)=\operatorname{det}\left(A C-B^{2}\right) \\
& =\operatorname{det} \tilde{D}(\lambda, q, k)=\left|\tilde{D}_{1} \tilde{D}_{2}^{*}+\tilde{D}_{+}^{2}-\tilde{D}_{-}^{2}\right|^{2}-4 \tilde{D}_{+}^{2} \operatorname{Re} \tilde{D}_{1} \operatorname{Re} \tilde{D}_{2} .
\end{aligned}
$$

Thanks to (9.30) we conclude that

$$
\lim _{\epsilon \rightarrow 0+} \epsilon^{-8} \tilde{\delta}_{\epsilon}(\lambda, \eta, \epsilon k)=\tilde{\delta}(\lambda, \eta, k)
$$

for each $\lambda>0$ and $(\eta, k) \in \mathbb{R}^{2}$.

The above, combined with (6.28), implies that for any $M>0$ and $\lambda_{0}$ as in Proposition 6.1 we have

$$
\tilde{\delta}(\lambda, \eta, k)>0, \quad|\eta|<M, \quad k \in \mathbb{R}, \quad \lambda>\lambda_{0} .
$$

The matrix $\tilde{D}(\lambda, \eta, k)$ is then invertible and, cf (6.38), $\tilde{D}^{-1}=\tilde{\delta}^{-1} \operatorname{adj}(\tilde{D})$. The adjugate of $\tilde{D}$ equals

$$
\operatorname{adj}(\tilde{D})=\left[\begin{array}{ll}
P & Q \\
Q & M
\end{array}\right],
$$

where $M, P$ and $Q$ are $2 \times 2$ matrices given by

$$
\begin{aligned}
& P:=\left[\begin{array}{ll}
\tilde{\mathrm{d}}_{1} & \tilde{\mathrm{d}}_{-} \\
\tilde{\mathrm{d}}_{-} & \tilde{\mathrm{d}}_{2}
\end{array}\right], \quad Q:=\left[\begin{array}{ll}
\left(\tilde{\mathrm{d}}_{+}\right)^{*} & \tilde{\mathrm{d}}_{o} \\
\tilde{\mathrm{d}}_{o} & \tilde{\mathrm{d}}_{+}
\end{array}\right], \\
& M:=\left[\begin{array}{ll}
\left(\tilde{\mathrm{d}}_{2}\right)^{*} & \left(\tilde{\mathrm{d}}_{-}\right)^{*} \\
\left(\tilde{\mathrm{d}}_{-}\right)^{*} & \left(\tilde{\mathrm{d}}_{1}\right)^{*}
\end{array}\right] .
\end{aligned}
$$


Here

$$
\begin{aligned}
\tilde{\mathrm{d}}_{1}:= & \left|\tilde{D}_{2}\right|^{2} \tilde{D}_{1}^{*}-\left(\tilde{D}_{+}^{2}+\tilde{D}_{-}^{2}\right) \operatorname{Re} \tilde{D}_{2}-i\left(\tilde{D}_{+}^{2}-\tilde{D}_{-}^{2}\right) \operatorname{Im} \tilde{D}_{2}, \\
\tilde{\mathrm{d}}_{2}:= & \left|\tilde{D}_{1}\right|^{2} \tilde{D}_{2}^{*}-\left(\tilde{D}_{+}^{2}+\tilde{D}_{-}^{2}\right) \operatorname{Re} \tilde{D}_{1}-i\left(\tilde{D}_{+}^{2}-\tilde{D}_{-}^{2}\right) \operatorname{Im} \tilde{D}_{1}, \\
\tilde{\mathrm{d}}_{-}:= & \tilde{D}_{+}\left(\tilde{D}_{+}^{2}-\tilde{D}_{-}^{2}\right)-\tilde{D}_{+} \tilde{D}_{1}^{*} \operatorname{Re} \tilde{D}_{2}+i \tilde{D}_{1}^{*} \tilde{D}_{+} \operatorname{Im} \tilde{D}_{2} \\
& =\tilde{D}_{+}\left(\tilde{D}_{+}^{2}-\tilde{D}_{-}^{2}\right)-\tilde{D}_{+} \tilde{D}_{2}^{*} \operatorname{Re} \tilde{D}_{1}+i \tilde{D}_{2}^{*} \tilde{D}_{+} \operatorname{Im} \tilde{D}_{1}, \\
\tilde{\mathrm{d}}_{+}:= & -\tilde{D}_{-}\left(\tilde{D}_{1} \tilde{D}_{2}^{*}+\tilde{D}_{+}^{2}-\tilde{D}_{-}^{2}\right), \\
\tilde{\mathrm{d}}_{o}:= & 2 \tilde{D}_{+} \tilde{D}_{-} \operatorname{Re} \tilde{D}_{2} .
\end{aligned}
$$

Thanks to (9.30) we conclude that

$$
\lim _{\epsilon \rightarrow 0+} \epsilon^{-6} \tilde{\mathrm{d}}_{j}^{(\epsilon)}(\lambda, \eta, \epsilon k)=\tilde{\mathrm{d}}_{j}(\lambda, \eta, k), \quad j \in\{-,+, 1,2, o\}
$$

for each $\lambda>0$ and $(\eta, k) \in \mathbb{R}^{2}$.

9.3.1. Limit of $V_{\epsilon, 1}^{(1)} \quad$ The limit in question is a special case of the following result.

Proposition 9.1. Suppose that $\varphi \in C^{2}(\mathbb{T})$ is such that $\varphi(0)=\varphi^{\prime}(0)=0$. Then, for any $M>0$ we have

$$
\lim _{\epsilon \rightarrow 0+} \int_{\mathbb{T}} \bar{w}_{\epsilon, \pm}^{(0)}(\lambda, \eta, k) \varphi(k) \mathrm{d} k=\frac{1}{2} \varphi^{\prime \prime}(0) \int_{\mathbb{R}} h^{2} w_{\phi, \pm}(\lambda, \eta, h) \mathrm{d} h
$$

and

$$
\lim _{\substack{\epsilon \rightarrow 0+\\ \lambda>\lambda_{0}}} \int_{\epsilon, \pm}^{(0)}(\lambda, \eta, k) \varphi(k) \mathrm{d} k=\frac{1}{2} \varphi^{\prime \prime}(0) \int_{\mathbb{R}} h^{2} y_{\phi, \pm}(\lambda, \eta, h) \mathrm{d} h, \quad|\eta| \leq M,
$$

Here $w_{\phi, \pm}$ and $y_{\phi, \pm}$ are given by (7.13).

Proof. We only prove (9.40), as the argument for (9.41) is very similar. The left hand side of (9.40) for $\bar{w}_{\epsilon,+}^{(0)}$ can be rewritten in the form

$$
\int_{\mathbb{T}} \tilde{\delta}_{\epsilon}^{-1}\left[\tilde{\mathrm{d}}_{1}^{(\epsilon)} \widehat{\bar{W}}_{\epsilon,+}+\tilde{\mathrm{d}}_{-}^{(\epsilon)} \widehat{\bar{Y}}_{\epsilon,+}+\left(\tilde{\mathrm{d}}_{+}^{(\epsilon)}\right)^{*} \widehat{\bar{Y}}_{\epsilon,-}+\tilde{\mathrm{d}}_{o}^{(\epsilon)} \widehat{\bar{W}}_{\epsilon,-}\right] \varphi(k) \mathrm{d} k .
$$

Denote by $J_{j, \epsilon}, j=1,2,3,4$ the respective terms arising after opening of the square bracket. Changing variables $k:=k / \epsilon$ we can write (cf (5.14))

$J_{1, \epsilon}=\frac{1}{2} \sum_{x, x^{\prime}} \int_{-1 /(2 \epsilon)}^{1 /(2 \epsilon)}\left(\tilde{\delta}_{\epsilon}^{-1} \tilde{d}_{1}^{(\epsilon)}\right)(\lambda, \eta, \epsilon k) \hat{\phi}^{*}\left(k+\frac{x}{\epsilon}-\frac{\eta}{2}\right) \hat{\phi}\left(k+\frac{x^{\prime}}{\epsilon}+\frac{\eta}{2}\right) \varphi(\epsilon k) \mathrm{d} k$.

Thanks to Lemma 8.1 we conclude that there exist $\lambda_{0}, \epsilon_{0}>0$ such that for any $\lambda>\lambda_{0}$ we have

$$
\sup _{\epsilon \in\left(0, \epsilon_{0}\right]} \sup _{k,|\eta| \leq M} R(\epsilon k)\left|\frac{\tilde{\mathrm{d}}_{l}^{(\epsilon)}(\lambda, \eta, \epsilon k)}{\tilde{\delta}_{\epsilon}(\lambda, \eta, \epsilon k)}\right|<+\infty .
$$


In addition, we have

$$
\sup _{\epsilon \in(0,1]|k| \leq 1 /(2 \epsilon)} \sup _{|\epsilon| k \mid)^{2}} \frac{(\epsilon k)}{R(\epsilon+\infty}
$$

Therefore,

$$
\sup _{\epsilon \in\left(0, \epsilon_{0}\right]} \sup _{k,|\eta| \leq M}|\varphi(\epsilon k)| \frac{\tilde{\mathrm{d}}_{l}^{(\epsilon)}(\lambda, \eta, \epsilon k)}{\tilde{\delta}_{\epsilon}(\lambda, \eta, \epsilon k)} \mid<+\infty .
$$

In fact, thanks to the rapid decay of the macroscopic wave function $\phi$, we can write

$$
\begin{aligned}
\lim _{\epsilon \rightarrow 0+} J_{1, \epsilon}= & \frac{1}{4} \lim _{\epsilon \rightarrow 0+} \int_{-1 /(2 \epsilon)}^{1 /(2 \epsilon)}\left(\tilde{\delta}_{\epsilon}^{-1} \tilde{\mathrm{d}}_{1}^{(\epsilon)}\right)(\lambda, \eta, \epsilon k) \hat{\phi}^{*}\left(k-\frac{\eta}{2}\right) \hat{\phi}\left(k+\frac{\eta}{2}\right) \\
& \times \epsilon^{2}\left(\varphi^{\prime \prime}(0) k^{2}+o(1)\right) \mathrm{d} k
\end{aligned}
$$

By virtue of the Lebesgue dominated convergence theorem, we conclude that the limit in (9.42) equals

$$
\frac{\varphi^{\prime \prime}(0)}{4} \int_{\mathbb{R}}\left(\tilde{\delta}^{-1} \tilde{\mathrm{d}}_{1}\right)(\lambda, \eta, h) h^{2} \hat{\phi}^{*}\left(h-\frac{\eta}{2}\right) \hat{\phi}\left(h+\frac{\eta}{2}\right) \mathrm{d} h .
$$

Dealing similarly with the remaining terms $J_{j, \epsilon}, j=2,3,4$ we conclude (9.40) for $\bar{w}_{\epsilon,+}^{(0)}$. The cases of $\bar{w}_{\epsilon,-}^{(0)}$ and $\bar{y}_{\epsilon, \pm}^{(0)}$ can be handled similarly.

Since $R(0)=R^{\prime}(0)=0$ and $R^{\prime \prime}(0)=12 \pi^{2}$ (cf (6.9) and (6.10)), by a direct application of Proposition 9.1, we obtain

$$
\lim _{\epsilon \rightarrow 0+} V_{\epsilon, 1}^{(1)}=8 \gamma \pi^{2} \int_{\mathbb{R}} h^{2} \mathfrak{w}_{\phi}(\lambda, \eta, h) \cdot \text { e d } h
$$

for all $|\eta| \leq M$ and $\lambda>\lambda_{0}$.

9.3.2. Limit of $V_{\epsilon, 1}^{(2)} \quad$ For any $J \in \mathcal{S}(\mathbb{R})$ such that $\hat{J}$ is supported in $[-M, M]$ we can write

$$
\begin{aligned}
& \int_{\mathbb{R}} V_{\epsilon, 1}^{(2)}(\lambda, \eta) \hat{J}^{*}(\eta) \mathrm{d} \eta \\
& \quad=\frac{2 \gamma}{3} \int_{\mathbb{R}} \int_{\mathbb{T}} \hat{J}^{*}\left\{\widehat{\widetilde{W}}_{\epsilon,+} \tilde{\Delta}_{1, \epsilon}+\widehat{\widetilde{Y}}_{\epsilon,+} \tilde{\Delta}_{2, \epsilon}+\widehat{\widetilde{Y}}_{\epsilon,-} \tilde{\Delta}_{2, \epsilon}^{*}+\widehat{\widetilde{W}}_{\epsilon,-} \tilde{\Delta}_{1, \epsilon}^{*}\right\} \frac{R \mathrm{~d} \eta \mathrm{d} k}{\tilde{\delta}_{\epsilon}} .
\end{aligned}
$$

By virtue of Lemma 8.1 we can use the Lebesgue dominated convergence theorem to enter with the limit, as $\epsilon \rightarrow 0+$, under the integral.

Combining (8.24) and (8.26) we conclude that

$$
\begin{aligned}
& \lim _{\epsilon \rightarrow 0+} \int_{\mathbb{R}} V_{\epsilon, 1}^{(2)} \hat{J}^{*}(\eta) \mathrm{d} \eta=\frac{2}{3} \lim _{\epsilon \rightarrow 0+} \int_{\mathbb{R}} \int_{\mathbb{T}} \hat{J}^{*}(\eta) \widehat{\widetilde{W}}_{\epsilon,+}(\eta, k) \mathrm{d} \eta \mathrm{d} k \\
& =\lim _{\epsilon \rightarrow 0+} \frac{\epsilon}{3} \sum_{x}\left|\tilde{\psi}_{x}^{(\epsilon)}\right|^{2} J(\epsilon x)=\frac{2}{3} \int_{\mathbb{R}} e_{\mathrm{th}}^{(0)}(y) J^{*}(y) \mathrm{d} y .
\end{aligned}
$$

The penultimate equality follows from (5.10). 


\section{Proof of Theorem 7.3}

\subsection{Proof of (7.17)}

Recall that $\tilde{\mathrm{d}}_{j}(\lambda, \eta, k), j \in\{1,2, o,-,+\}$ and $\operatorname{det} \tilde{D}(\lambda, \eta, k)$ are given by (9.38) and (9.34) respectively. We recall also that $\tilde{\Delta}_{j}(\lambda, q, k), j=1,2$ are defined by a modification of formulas (7.5) where the coefficients $\tilde{\mathrm{d}}_{i}^{(\epsilon)}$ have been replaced by the corresponding $\tilde{\mathrm{d}}_{\iota}$.

Given $\varphi \in C(\mathbb{T})$ we can write

$$
\int_{\mathbb{T}} w_{\epsilon,+}(\lambda, \eta, k) \varphi(k) \mathrm{d} k=\int_{\mathbb{T}}\left(I_{\epsilon}+I I_{\epsilon}+I I I_{\epsilon}+I V_{\epsilon}\right) \varphi(k) \mathrm{d} k
$$

Here $I_{\epsilon}, I I_{\epsilon}, I I I_{\epsilon}, I V_{\epsilon}$ are given by (8.23). By virtue of (8.25) we conclude that

$$
\lim _{\epsilon \rightarrow 0+} \int_{\mathbb{T}} I_{\epsilon} \varphi(k) \mathrm{d} k=\lim _{\epsilon \rightarrow 0+} w_{\epsilon}^{(-)} \int_{\mathbb{T}} \varphi(k) \mathrm{d} k, \quad|\eta| \leq M, \quad \lambda>\lambda_{0} .
$$

Lemma 10.1. For any $M>0$

$$
\lim _{\epsilon \rightarrow 0+} \int_{\mathbb{T}}\left|I V_{\epsilon}\right| \mathrm{d} k=0, \quad|\eta| \leq M, \lambda>\lambda_{0} .
$$

Proof. Note that, according to Proposition 6.1, for each $M>0$ we can choose $\lambda_{0}, \epsilon_{0}>0$ such that for $\lambda>\lambda_{0}$

$$
\begin{gathered}
\int_{\mathbb{T}}\left|I V_{\epsilon}(\lambda, \eta, k)\right| \mathrm{d} k \leq \epsilon^{3}\left(\sum_{j=1}^{4}\left\|r_{\epsilon}^{(j)}\right\|_{\mathcal{A}^{\prime}}\right) \sup _{k} \tilde{\delta}_{\epsilon}^{-1}\left(\sum_{j}\left|\tilde{\mathrm{d}}_{j}^{(\epsilon)}\right|\right) \\
\preceq \epsilon^{3}\left(\sum_{j=1}^{4}\left\|r_{\epsilon}^{(j)}\right\|_{\mathcal{A}^{\prime}}\right) \sup _{k}\left(\tilde{\delta}_{\epsilon}^{(0)}\right)^{-1}\left(\sum_{j}\left|\tilde{\mathrm{d}}_{j}^{(\epsilon)}\right|\right)
\end{gathered}
$$

for $|\eta| \leq M$ and $\epsilon \in\left(0, \epsilon_{0}\right]$. Thanks to (8.5) we conclude that

$$
\epsilon^{3}\left(\tilde{\delta}_{\epsilon}^{(0)}\right)^{-1}\left|\tilde{\mathrm{d}}_{-}^{(\epsilon)}\right| \preceq \epsilon^{3}\left(\tilde{\delta}_{\epsilon}^{(0)}\right)^{-1}\left(\sum_{j=0}^{3} R_{\epsilon}^{3-j}\left(\lambda \epsilon^{2}\right)^{j}\right) .
$$

Invoking the definition of $\tilde{\delta}_{\epsilon}^{(0)}$, see (6.27), we can bound the right hand side of (10.3) by $\epsilon^{3}\left(R_{\epsilon}+\lambda \epsilon^{2}\right)^{-1} \preceq \epsilon$. The conclusion of the lemma follows then directly from the above estimate and (6.18). 
Using a similar argument we infer that for any $\lambda>\lambda_{0},|\eta| \leq M$ we have

$$
\begin{array}{r}
\sup _{k}\left|I I_{\epsilon}\right| \preceq \epsilon_{k}^{2} \sup _{\epsilon} \tilde{\delta}_{\epsilon}^{-1}\left(\sum_{j}\left|\tilde{\mathrm{d}}_{j}^{(\epsilon)}\right|\right) \\
\preceq \epsilon_{k}^{2} \sup _{k}\left(\tilde{\delta}_{\epsilon}^{(0)}\right)^{-1}\left(\sum_{j}\left|\tilde{\mathrm{d}}_{j}^{(\epsilon)}\right|\right) \preceq 1
\end{array}
$$

for $\epsilon \in\left(0, \epsilon_{0}\right]$, thanks to (8.5) and (6.27). On the other hand, due to Proposition 6.2 , for any $\lambda>\lambda_{0}, k \neq 0$ and $|\eta| \leq M$ we have

$$
\lim _{\epsilon \rightarrow 0+} \epsilon^{2} \tilde{\delta}_{\epsilon}^{-1}(\lambda, \eta, k)\left(\sum_{j}\left|\tilde{\mathrm{d}}_{j}^{(\epsilon)}(\lambda, \eta, k)\right|\right)=0
$$

By virtue of the Lebesgue dominated convergence theorem we conclude therefore that

$$
\lim _{\epsilon \rightarrow 0+} \int_{\mathbb{T}} I I_{\epsilon} \varphi(k) d k=0
$$

Finally, we have

$\int_{\mathbb{T}} I I I_{\epsilon} \varphi(k) \mathrm{d} k=\epsilon^{2} \int_{\mathbb{T}} \tilde{\delta}_{\epsilon}^{-1}\left\{\widehat{W}_{\epsilon,+} \tilde{\mathrm{d}}_{1}^{(\epsilon)}+\widehat{Y}_{\epsilon,+} \tilde{\mathrm{d}}_{-}^{(\epsilon)}+\widehat{Y}_{\epsilon,-}\left(\tilde{\mathrm{d}}_{+}^{(\epsilon)}\right)^{*}+\widehat{W}_{\epsilon,-} \tilde{\mathrm{d}}_{o}^{(\epsilon)}\right\} \varphi(k) \mathrm{d} k$.

The computation of the limit, as $\epsilon \rightarrow 0+$, of each of the four expressions $J_{j}^{(\epsilon)}$, $j=1, \ldots, 4$ that arise in the right hand side after opening of the bracket is almost identical so we explain only how to deal with the first one. Using (5.14) we can write that $J_{1}^{(\epsilon)}=\sum_{j=1}^{2} J_{1 j}^{(\epsilon)}$, with (cf (5.14))

$$
\begin{aligned}
& J_{11}^{(\epsilon)}:=\epsilon^{2} \int_{\mathbb{T}} \delta_{\epsilon}^{-1} \tilde{d}_{1}^{(\epsilon)} \widehat{\bar{W}}_{\epsilon,+}(\eta, k) \varphi(k) \mathrm{d} k, \\
& J_{12}^{(\epsilon)}:=\epsilon^{2} \int_{\mathbb{T}}\left(\tilde{\delta}_{\epsilon}^{-1} \tilde{d}_{1}^{(\epsilon)}\right) \widehat{\widetilde{W}}_{\epsilon,+}(\eta, k) \varphi(k) \mathrm{d} k .
\end{aligned}
$$

In what follows we show that

$$
\lim _{\epsilon \rightarrow 0+} J_{12}^{(\epsilon)}=0
$$

and, cf (7.14),

$$
\lim _{\epsilon \rightarrow 0+} J_{1}^{(\epsilon)}=\lim _{\epsilon \rightarrow 0+} J_{11}^{(\epsilon)}=\varphi(0) \int_{\mathbb{R}} \frac{\widehat{W}_{\phi,+}(0) \tilde{\mathrm{d}}_{1}}{\operatorname{det} \tilde{D}} \mathrm{~d} k .
$$


We repeat the above argument to compute the limits of the remaining terms $J_{j}^{(\epsilon)}$ and obtain that, $\mathrm{cf}(9.31)$

$$
\begin{aligned}
& \lim _{\epsilon \rightarrow 0+} \int_{\mathbb{T}} I I I_{\epsilon} \varphi(k) \mathrm{d} k \\
& =\varphi(0) \int_{\mathbb{R}} \operatorname{det} \tilde{D}^{-1}\left\{\widehat{W}_{\phi,+}(0) \tilde{\mathrm{d}}_{1}+\widehat{Y}_{\phi,+}(0) \tilde{\mathrm{d}}_{-}+\widehat{Y}_{\phi,-}(0)\left(\tilde{\mathrm{d}}_{+}\right)^{*}+\widehat{W}_{\phi,-}(0) \tilde{\mathrm{d}}_{o}\right\} \mathrm{d} h \\
& =\varphi(0) \int_{\mathbb{R}} w_{\phi,+}(\lambda, \eta, h) \mathrm{d} h .
\end{aligned}
$$

10.1.1. Proof of (10.8) After the change of variables $k^{\prime}:=k / \epsilon$ we can write

$$
J_{1}^{(\epsilon)}=\sum_{x, x^{\prime}} \int_{-1 /(2 \epsilon)}^{1 /(2 \epsilon)} \frac{\epsilon^{-6} \tilde{\mathrm{d}}_{1}^{(\epsilon)}(\lambda, \eta, \epsilon k)}{\epsilon^{-8} \tilde{\delta}_{\epsilon}(\lambda, \eta, \epsilon k)} \hat{\phi}^{*}\left(\frac{x}{\epsilon}+k-\frac{\eta}{2}\right) \hat{\phi}\left(\frac{x^{\prime}}{\epsilon}+k+\frac{\eta}{2}\right) \varphi(\epsilon k) \mathrm{d} k .
$$

Using the argument from the proof of Lemma 10.1 we conclude that for any $M>0$ and $\lambda>\lambda_{0}$, where $\lambda_{0}, \epsilon_{0}>0$ are as in the statement of Proposition 6.1,

$$
\left|\frac{\epsilon^{-6} \tilde{\mathrm{d}}_{1}^{(\epsilon)}(\lambda, \eta, \epsilon k)}{\epsilon^{-8} \tilde{\delta}_{\epsilon}(\lambda, \eta, \epsilon k)}\right| \preceq 1
$$

for all $k \in \mathbb{R},|\eta| \leq M$ and $\epsilon \in\left(0, \epsilon_{0}\right]$. Due to the decay of the wave function $\hat{\phi}$ we conclude that

$$
\lim _{\epsilon \rightarrow 0+} J_{1}^{(\epsilon)}=\lim _{\epsilon \rightarrow 0+} \int_{-1 /(2 \epsilon)}^{1 /(2 \epsilon)} \frac{\epsilon^{-6} \tilde{\mathrm{d}}_{1}^{(\epsilon)}(\lambda, \eta, \epsilon k)}{\epsilon^{-8} \tilde{\delta}_{\epsilon}(\lambda, \eta, \epsilon k)} \hat{\phi}^{*}\left(k-\frac{\eta}{2}\right) \hat{\phi}\left(k+\frac{\eta}{2}\right) \varphi(\epsilon k) \mathrm{d} k .
$$

Thanks to (10.11) to compute the last limit we can use the Lebesgue dominated convergence and conclude, using (9.35) and (9.39), that the right hand side of the above equality coincides with the right hand side of (10.8).

10.1.2. Proof of (10.7) Using condition (2.12) we conclude that for some $r>1$

$$
\sup _{\eta} \int_{\mathbb{T}}\left|\widehat{\widetilde{W}}_{\epsilon,+}(\eta, k)\right|^{r} \mathrm{~d} k<+\infty
$$

Combining the above with estimate (10.4) together with the limit (10.5) we conclude that for any $\lambda>\lambda_{0}$ and $|\eta| \leq M$

$$
\lim _{\epsilon \rightarrow 0+} \epsilon^{2} \int_{\mathbb{T}}\left|\widehat{\widetilde{W}}_{\epsilon,+}(\eta, k) \varphi(k)\right| \frac{\tilde{\mathrm{d}}_{1}^{(\epsilon)}(\lambda, \eta, k)}{\tilde{\delta}_{\epsilon}(\lambda, \eta, k)} \mid \mathrm{d} k=0
$$

This obviously implies (10.7). 


\subsection{Proof of (7.18)}

We use the notation from Section 8.4 and carry out our analysis only for $y_{\epsilon,+}$, as the argument for $y_{\epsilon,-}$ is very similar. For any $\varphi \in C(\mathbb{T})$ we have

$$
\int_{\mathbb{T}} y_{\epsilon}(\lambda, \eta, k) \varphi \mathrm{d} k=\int_{\mathbb{T}} I_{\epsilon} \varphi \mathrm{d} k+\int_{\mathbb{T}} I I_{\epsilon} \varphi \mathrm{d} k+\int_{\mathbb{T}} I I I_{\epsilon} \varphi \mathrm{d} k+\int_{\mathbb{T}} I V_{\epsilon} \varphi \mathrm{d} k .
$$

The analysis of the terms on the right hand side of (10.13) is very similar to the one done in Section 10.2. As a result we obtain

$$
\lim _{\epsilon \rightarrow 0+} \int_{\mathbb{T}}\left(\left|I_{\epsilon}\right|+\left|I I I_{\epsilon}\right|+\left|I V_{\epsilon}\right|\right) \mathrm{d} k=0
$$

In addition,

$$
\begin{aligned}
& \lim _{\epsilon \rightarrow 0+} \int_{\mathbb{T}} I I_{\epsilon} \mathrm{d} k \\
& =\varphi(0) \int_{\mathbb{R}} \operatorname{det} \tilde{D}^{-1}\left\{\widehat{W}_{\phi,+} \tilde{\mathrm{d}}_{-}+\widehat{Y}_{\phi,+} \tilde{\mathrm{d}}_{2}+\widehat{Y}_{\phi,-}\left(\tilde{\mathrm{d}}_{o}\right)^{*}+\widehat{W}_{\phi,-} \tilde{\mathrm{d}}_{-}\right\} d h \\
& =\varphi(0) \int_{\mathbb{R}} y_{\phi,+}(\lambda, \eta, h) \mathrm{d} h
\end{aligned}
$$

and (7.18) follows.

\section{Proof of Theorem 3.1}

Suppose that $\kappa(t, y)$ and $p(t, y)$ satisfy (3.1). Then, $\hat{\phi}(t, h)-$ the Fourier transform of

$$
\phi(t, y):=\frac{\tau_{2}}{4} \kappa(t, y)+i p(t, y)
$$

satisfies

$$
\frac{\mathrm{d}}{\mathrm{d} t} \hat{\phi}(t, h)=-i \tau_{2}(\pi h)^{2} \hat{\phi}(t, h)-6 \gamma \pi^{2} h^{2}\left[\hat{\phi}(t, h)-(\hat{\phi})^{*}(t,-h)\right] .
$$

Let

$$
\hat{\bar{\psi}}_{\epsilon}(t, k):=\epsilon \mathbb{E}_{\epsilon} \hat{\psi}^{(\epsilon)}(t, \epsilon k), \quad k \in \epsilon^{-1} \mathbb{T} .
$$

From (5.19) we obtain

$$
\frac{\mathrm{d}}{\mathrm{d} t} \hat{\bar{\psi}}_{\epsilon}(t, k)=\frac{-i \omega(\epsilon k)}{\epsilon^{2}} \hat{\bar{\psi}}_{\epsilon}(t, k)-\frac{\gamma R(\epsilon k)}{\epsilon^{2}}\left[\hat{\bar{\psi}}_{\epsilon}(t, k)-\left(\hat{\bar{\psi}}_{\epsilon}\right)^{*}(t,-k)\right] .
$$

After a straightforward calculation we obtain, using (2.13) that for any $G \in C_{0}^{\infty}(\mathbb{R})$

$$
\int_{\mathbb{R}} G^{*}(y) \phi(y) \mathrm{d} y=\lim _{\epsilon \rightarrow 0+} \epsilon \sum_{x} G^{*}(\epsilon x)\left\langle\psi_{x}\right\rangle_{\mu_{\epsilon}}=\lim _{\epsilon \rightarrow 0+} \int_{\epsilon^{-1} \mathbb{T}} \hat{G}^{*}(k) \hat{\bar{\psi}}_{\epsilon}(0,-k) \mathrm{d} k .
$$


Since

$$
\lim _{\epsilon \rightarrow 0+} \frac{\omega(\epsilon k)}{\epsilon^{2}}=\tau_{2} \pi^{2} k^{2} \text { and } \frac{R(\epsilon k)}{\epsilon^{2}}=6 \pi^{2} k^{2},
$$

uniformly on compact intervals, an elementary stability theory for solutions of ordinary differential equations guarantees that for any $T, M>0$ we have

$$
\hat{\bar{\psi}}_{\epsilon}(t, k)=(1+o(1)) \hat{\bar{\psi}}_{\epsilon}^{(0)}(t, k)
$$

uniformly on $|k| \leq M,|t| \leq T$, as $\epsilon \ll 1$, where $\hat{\bar{\psi}}_{\epsilon}^{(0)}(0, k)$ satisfies (11.1) with the initial condition $\hat{\bar{\psi}}_{\epsilon}^{(0)}(0, k):=\hat{\bar{\psi}}_{\epsilon}(0, k)$. Equation (11.1) can be solved explicitly. Taking into account (11.3) we obtain, upon letting $\epsilon \rightarrow 0+$, that

$$
\lim _{\epsilon \rightarrow 0+} \int_{\epsilon^{-1} \mathbb{T}} \hat{G}^{*}(k) \hat{\bar{\psi}}_{\epsilon}^{(0)}(t,-k) \mathrm{d} k=\int_{\mathbb{R}} \hat{G}^{*}(h) \hat{\phi}(t,-h) \mathrm{d} h,
$$

where $\hat{\bar{\psi}}(t, h)$ satisfies (11.1) with the initial $\hat{\bar{\psi}}(0, h):=\hat{\phi}(0, h)$. Therefore $\hat{\bar{\psi}}(t, h)=\hat{\phi}(t, h)$ and, in conclusion,

$$
\begin{aligned}
& \lim _{\epsilon \rightarrow 0+} \epsilon \sum_{x} G^{*}(\epsilon x) \mathbb{E}_{\epsilon} \psi_{x}^{(\epsilon)}(t)=\lim _{\epsilon \rightarrow 0+} \int_{\epsilon^{-1} \mathbb{T}} \hat{G}^{*}(k) \hat{\bar{\psi}}_{\epsilon}(t,-k) \mathrm{d} k \\
&=\int_{\mathbb{R}} \hat{G}^{*}(h) \hat{\phi}(t,-h) \mathrm{d} h=\int_{\mathbb{R}} G^{*}(y) \phi(t, y) \mathrm{d} y .
\end{aligned}
$$

Open Access This article is distributed under the terms of the Creative Commons Attribution 4.0 International License (http://creativecommons.org/licenses/by/4.0/), which permits unrestricted use, distribution, and reproduction in any medium, provided you give appropriate credit to the original author(s) and the source, provide a link to the Creative Commons license, and indicate if changes were made.

\section{References}

1. Basile, G., Bernardin, C., Olla, S.: A momentum conserving model with anomalous thermal conductivity in low dimension. Phys. Rev. Lett. 96, 204303, 2006. doi:10.1103/ PhysRevLett.96.204303

2. Basile, G., Bernardin, C., Olla, S.: Thermal conductivity for a momentum conservative model. Commun. Math. Phys. 287, 67-98, 2009

3. Basile, G., Olla, S., Spohn, H.: Energy transport in stochastically perturbed lattice dynamics. Arch. Ration. Mech. 195(1), 171-203, 2009

4. Da Prato, G., Zabczyk, J.: Stochastic Equations in Infinite Dimensions. Cambridge Univ. Press, Cambridge, 1992

5. Eidelman, S.D.: Parabolic systems (in Russian). Nauka, 1964

6. Even, N., Olla, S.: Hydrodynamic Limit for an Hamiltonian System with Boundary Conditions and Conservative Noise. Arch. Rational Mech. Anal. 213, 561-585, 2014

7. Gantmakher, F.R.: The Theory of Matrices, vol. 1. AMS Chelsea 2000 
8. Jara, M., Komorowski, T., Olla, S.: Superdiffusion of energy in a chain of harmonic oscillators with noise. Commun. Math. Phys. 339, 407-453, 2015

9. Komorowski, T., Olla, S.: Ballistic and superdiffusive scales in macroscopic evolution of a chain of oscillators. Nonlinearity 29, 962-999, 2016

10. LePri, S., Livi, R., Politi, A.: Thermal Conduction in classical low-dimensional lattices. Phys. Rep. 377, 1-80, 2003

11. Lepri, S., Livi, R., Politi, A.: Heat conduction in chains of nonlinear oscillators. Phys. Rev. Lett. 78, 1896, 1997

12. Spohn, H.: Nonlinear fluctuating hydrodynamics for anharmonic chains. J. Stat. Phys. 154(5), 1191-1227, 2014

13. Varadhan, S.R.S.: Nonlinear diffusion limit for a system with nearest neighbor interactions II. In: Asymptotic Problems in Probability Theory, Stochastic Models and Diffusions on Fractals. Pitman Research Notes in Mathematics Series, vol. 283, pp. 75128 (1994)

\author{
Institute of Mathematics, \\ Polish Academy of Sciences \\ Warsaw, Poland. \\ e-mail: komorow@ hektor.umcs.lublin.pl \\ and \\ CNRS, CEREMADE,Université Paris Dauphine, \\ PSL Research University, \\ 75016 Paris, France. \\ e-mail: olla@ ceremade.dauphine.fr
}

(Received January 29, 2016 / Accepted July 22, 2016)

Published online August 2, 2016 - (C) The Author(s) (2016)

This article is published with open access at Springerlink.com 